\title{
Charge Transfer Reactions
}

\author{
Konrad Dennerl
}

Received: 29 July 2010 / Accepted: 15 November 2010 / Published online: 11 December 2010

(C) The Author(s) 2010. This article is published with open access at Springerlink.com

\begin{abstract}
Charge transfer, or charge exchange, describes a process in which an ion takes one or more electrons from another atom. Investigations of this fundamental process have accompanied atomic physics from its very beginning, and have been extended to astrophysical scenarios already many decades ago. Yet one important aspect of this process, i.e. its high efficiency in generating X-rays, was only revealed in 1996, when comets were discovered as a new class of X-ray sources. This finding has opened up an entirely new field of $\mathrm{X}$-ray studies, with great impact due to the richness of the underlying atomic physics, as the $\mathrm{X}$-rays are not generated by hot electrons, but by ions picking up electrons from cold gas. While comets still represent the best astrophysical laboratory for investigating the physics of charge transfer, various studies have already spotted a variety of other astrophysical locations, within and beyond our solar system, where X-rays may be generated by this process. They range from planetary atmospheres, the heliosphere, the interstellar medium and stars to galaxies and clusters of galaxies, where charge transfer may even be observationally linked to dark matter. This review attempts to put the various aspects of the study of charge transfer reactions into a broader historical context, with special emphasis on X-ray astrophysics, where the discovery of cometary X-ray emission may have stimulated a novel look at our universe.
\end{abstract}

Keywords Charge transfer $\cdot$ Charge exchange $\cdot$ Atomic physics $\cdot \mathrm{X}$-rays $\cdot$ Ions $\cdot$ Energetic neutral atoms $\cdot$ Nuclear fusion $\cdot$ X-ray astronomy $\cdot$ History $\cdot$ Solar wind $\cdot$ Comets $\cdot$ Moon $\cdot$ Planets · Venus · Earth · Mars · Jupiter · Saturn · Heliosphere $\cdot$ X-ray background · Local bubble $\cdot$ Interstellar medium $\cdot$ North polar spur $\cdot$ Supernova remnants $\cdot$ Stars $\cdot$ Galaxies $\cdot$ Clusters of galaxies · Dark matter · Rosat · EUVE - BeppoSAX · Chandra ·

XMM-Newton $\cdot$ Suzaku $\cdot$ ASTRO-H $\cdot$ IXO

K. Dennerl ( $\varangle)$

Max-Planck-Institut für extraterrestrische Physik, Giessenbachstraße, 85748 Garching, Germany

e-mail: kod@mpe.mpg.de 


\section{Introduction}

Charge transfer is a fundamental process in atomic physics which has been studied in various contexts for a long time. During the last decade, it has received renewed attention, when the discovery of cometary X-ray emission revealed that this process can be an important source of X-ray emission, which was overlooked before. Since this discovery (announced on April 4, 1996; Lisse et al. 1996a) and its correct interpretation as the direct result of charge transfer between highly charged heavy ions in the solar wind and cometary neutrals (Cravens 1997), investigations on charge transfer reactions have expanded considerably, comprising experimental studies in the laboratory and theoretical calculations of the underlying atomic physics as well as astrophysical studies, which are not restricted to comets anymore but cover a wide range, from planetary atmospheres to clusters of galaxies. The fact that X-rays are not the result of excitation by hot electrons, but by ions picking up electrons from cold gas has opened up an entirely new field of X-ray studies, which is likely to continue to grow.

In view of this exciting development it may be now, 14 years after the discovery of cometary X-ray emission, a good time for a broad review of the history and current status of charge transfer studies and their various implications. Specific aspects of this topic have already been addressed in earlier reviews: Krasnopolsky et al. (2004) and Lisse et al. (2004) reviewed charge transfer reactions in the context of the X-ray and EUV emission from comets. Krasnopolsky et al. (2004) discussed also alternative excitation processes and presented detailed discussions of theoretical modeling methods, spectra, and an extensive list of relevant laboratory measurements of charge transfer reactions. Bhardwaj et al. (2007) reviewed the various processes which give rise to X-rays from solar system objects, including charge transfer reactions. Wargelin et al. (2008) presented a brief review of the astrophysical relevance of charge transfer reactions in the solar system and beyond, and focused on charge exchange spectra, experimental approaches, and theoretical modeling efforts.

This review attempts to provide a concise overview on the various aspects of charge transfer reactions in a historical context, with specific emphasis on X-ray astrophysics. It is outlined in the following way: In Sect. 2, a short overview about the physics of charge transfer and its investigations by theoretical studies, laboratory experiments, and astrophysical observations, will be given. Section 3 will cover the history of charge transfer studies, from its very beginnings to the present, and the next two sections will summarize our current knowledge about the astrophysical environments where charge transfer reactions are known or expected to take place, both in the solar system (Sect. 4) and beyond (Sect. 5). The paper will close with conclusions and a short outlook in Sect. 6.

An alternative name for charge transfer is charge exchange. Both notations will be adopted in this paper, with some preference for the designation which appears to be more common in the corresponding context. For brevity, the abbreviation $C X E$ will be used for Charge Exchange (or Charge Transfer) induced $\mathbf{X}$-ray Emission.

\section{Physics of Charge Transfer}

In its basic principle, charge transfer is a very simple process: charge (in the form of one or more electrons) is transferred from an atom or molecule to an ion that gets into an excited state, from which it may deexcite by releasing electromagnetic radiation. If the initial charge of the ion was sufficiently high, then the energy of the radiation can be in the X-ray range. The main characteristics of charge transfer are a very high cross section (of the order of $10^{-15} \mathrm{~cm}^{2}$ ) and characteristic line emission at X-ray energies and below. Despite its apparent simplicity, however, a detailed understanding of this fundamental process is a most challenging task. In the following, various investigation methods will be briefly summarized. 


\subsection{Theoretical Studies of Charge Transfer}

A detailed theoretical description of charge transfer is so challenging that several approximative techniques have been developed. In the following, their main characteristics are briefly listed, according to Hasan et al. (2001). The most elementary approach is the classical overthe-barrier (COB) model (Ryufuku et al. 1980), which gives an estimate of the capture cross section into the classically most likely $n$-level, but does not predict the distribution of $n$ - and $\ell$-levels. A more sophisticated approach, which takes the three-body dynamics between the projectile ion, the target ion, and one active target electron into account, is the classical trajectory Monte Carlo (CTMC) technique (Cornelius et al. 2000). This method mimics certain quantum characteristics by choosing an ensemble of projectile-target configurations, computed by numerically solving Hamiltons equations for a mutually interacting three-body system, and propagates them by classical mechanics in order to simulate the collision. However, it neglects the detailed molecular states associated to the vibrationally excited levels of the target (Otranto et al. 2006) and is of limited use at energies below $1 \mathrm{keV} / \mathrm{amu}$, where the structure of the projectile ion is important (Kallman and Palmeri 2007). ${ }^{1}$ Another approach, the Landau-Zener (LZ) approximation, can often elucidate the general behaviour of the cross sections and can be adopted for estimating the state-selective capture, but is of limited accuracy. The most reliable theoretical approach is the quantum-mechanical close-coupling (CC) method, which treats the relevant atomic and/or quasi-molecular structure and reaction dynamics, but this method is very challenging in terms of technique and computational resources.

The methods listed above are typically applied to single electron capture (SEC). While this is usually the most important capture channel in slow collisions of multiply charged ions with atoms and molecules, multi-electron capture (MEC) can be collectively as important and sometimes even more important than SEC (Ali et al. 1994; Hasan et al. 2001; Rigazio et al. 2002; Otranto et al. 2006). The challenges for a correct theoretical treatment of MEC, however, are even higher than for SEC. Another important aspect for a realistic theoretical description is to take the variation of $n$ - and $\ell$-levels of the captured electron with the collision velocity into account, as well as other reaction channels, like dissociative and nondissociative, Auger and radiative relaxation (Hasan et al. 2001).

\subsection{Laboratory Studies of Charge Transfer}

While laboratory studies of the charge transfer process have been performed for many decades (Sect. 3.1; for a concise overview, see also Kallman and Palmeri 2007), the discovery of cometary X-ray emission has intensified this kind of research in recent years. Laboratory-based investigations have been performed by at least four independent groups (e.g. Otranto and Olson 2008):

- the Jet Propulsion Laboratory (JPL), focusing on the determination of total cross sections for highly charged $\mathrm{C}, \mathrm{N}, \mathrm{O}$, and $\mathrm{Ne}$ ions interacting with $\mathrm{H}_{2} \mathrm{O}, \mathrm{He}, \mathrm{H}_{2}$, and $\mathrm{CO}_{2}$ at typical solar wind velocities (Greenwood et al. 2000, 2001)

- the Lawrence Livermore National Laboratory (LLNL), using an Electron Beam Ion Trap (EBIT) with a microcalorimeter detector in order to obtain very high resolution X-ray data, though at lower collision energies, from stationary ions which result from charge

\footnotetext{
${ }^{1}$ For solar wind ions, the energy range of interest is between $\sim 0.8 \mathrm{keV} / \mathrm{amu}$ and $\sim 3.0 \mathrm{keV} / \mathrm{amu}$ for the slow and fast solar wind.
} 
exchange of various ions, e.g., $\mathrm{O}^{8+}$, with $\mathrm{CO}_{2}, \mathrm{CH}_{4}, \mathrm{~N}_{2}, \mathrm{He}$, and alcohol (Beiersdorfer et al. 2000, 2001, 2003, Wargelin et al. 2005, 2008)

- at KVI Groningen, investigating collisions that emit photons in the EUV range, such as $\mathrm{He}^{2+}$ on $\mathrm{H}_{2} \mathrm{O}, \mathrm{CO}, \mathrm{CO}_{2}, \mathrm{CH}_{4}$, and $\mathrm{O}^{6+}$ on $\mathrm{H}_{2} \mathrm{O}$ (Bodewits et al. 2006; Bodewits and Hoekstra 2007)

- at Belfast, focusing on $\mathrm{O}^{6+}$ collisions with $\mathrm{H}_{2} \mathrm{O}, \mathrm{CO}_{2}, \mathrm{CH}_{4}$, and $\mathrm{C}^{4+}$ on $\mathrm{CH}_{4}$ by means of the Translational Energy Spectroscopy (TES) technique (Seredyuk et al. 2005)

- in addition, several groups have used the Cold Target Recoil Ion Momentum Spectroscopy (COLTRIMS) technique (Dörner et al. 2000) to obtain state-selective charge exchange cross sections (e.g., Chesnel et al. 1998; Hasan et al. 2001; Edgu-Fry et al. 2004).

Mawhorter et al. (2007) find that the CTMC calculations yield total single electron capture cross sections which are consistently smaller than the measurements by typically $15-50 \%$. They note that one source of the discrepancy might be the presence of autoionizing multiple electron captures to adjacent energy levels in present experiments, which leave just one single electron captured and hence contribute to the true single capture cross section, while CTMC calculations include only the direct single electron capture. Another source of the offset, assuming that the classical nature of CTMC is not the limiting factor at these collision energies, my lie in the assumption that there is only one active electron.

Despite all the efforts in laboratory studies of the charge transfer process, there are, however, also general limitations of this approach. Some of the emitted photons from the metastable state, e.g., may not be detected in neutral-ion-beam experiments: due to the long lifetime of $\sim 10^{-3} \mathrm{~s}$ of the $\mathrm{O}^{\star 6+}\left(2^{3} S\right)$ state, metastable ions with a velocity of $400 \mathrm{~km} / \mathrm{s}$ move about $400 \mathrm{~m}$ without any emission (Kharchenko 2005). This problem is avoided by EBIT experiments, where the ions are trapped. These experiments, however, collect only spectra and cannot measure absolute charge exchange cross sections.

\subsection{Astrophysical Studies of Charge Transfer with Comets}

In the year 1996 (Sect. 3.2) a new window opened, offering a third option for the study of the physics of charge transfer: by observing the X-ray emission of comets (Lisse et al. 1996b; Dennerl et al. 1997; Mumma et al. 1997). This emission is the direct result of charge transfer between highly charged heavy ions in the solar wind and cometary neutrals (Cravens 1997). As the gas around comets is cold, there is essentially no thermal X-ray emission, and as it is not subject to a strong electric or magnetic field, X-ray emission by energetic electrons is negligible. ${ }^{2}$ Furthermore, the cometary nucleus is so small, and the gas and the embedded dust grains are so diluted, that there are not enough targets for solar X-ray scattering to become important. ${ }^{3}$ This has the exciting consequence that the X-ray emission of comets is essentially pure CXE.

\footnotetext{
${ }^{2}$ The idea that X-rays might be generated by energetic electrons was the motivation for the first (unsuccessful) X-ray observation of a comet, with the Einstein satellite (Hudson et al. 1991), and it was among the early suggestions for explaining the X-ray emission of comets detected since 1996 (Bingham et al. 1997, 1998; Northrop et al. 1997; Northrop 1997; Uchida et al. 1998; Shapiro et al. 1999; Torney et al. 2001). However, energetic electrons were found to contribute only $0.025 \%$ (Wegmann et al. 1998) or 0.2\%-4\% (Krasnopolsky 2001) to the observed X-ray and EUV emission.

${ }^{3}$ Also dust grains were considered as a possible origin of the X-ray emission, either by collisions with interplanetary dust (Ibadov 1990, 1996) or themselves (Ip and Chow 1997), or by scattering of solar X-rays (Wickramasinghe and Hoyle 1996). However, none of these possibilities could be unambiguously proven to date.
} 
Thus, comets represent perhaps the best laboratory for studying the physics of charge transfer. With highly charged heavy ions streaming into the cold cometary gas, nature is providing a clean experimental setup and a textbook example of a system which is far away from thermal equilibrium. For observing such a system, the full range of capabilities of current X-ray instrumentation, concerning their spatial, temporal, and spectral resolution, can be utilized: the cometary X-ray emission is so extended that it can be spatially resolved, allowing studies of its morphology, the temporal resolution makes it possible to correct for the apparent motion and to investigate the temporal variability of the X-ray signal, and spectral resolution is the key for revealing the elusive properties of charge transfer.

Like the theoretical or laboratory approach, this third option for the study of charge transfer, by using X-ray observations of comets, is not free from challenges either. Comets are moving targets, exhibiting extended X-ray emission which may fill or even overfill the field of view. Therefore, the discrimination of the cometary signal from other celestial X-ray sources, in particular from the diffuse soft X-ray background, is a nontrivial task, as well as discrimination from the detector noise of X-ray CCDs, which may contribute significantly to the total count rate at the lowest energies. The other challenge is the spectral modeling, which requires an accurate knowledge of the detector response at energies below $\sim 1 \mathrm{keV}$, where most of the cometary X-ray emission is observed. This, however, is also the most difficult spectral region to calibrate, because there the distortion of spectral lines is highest, and because there are not many celestial sources for providing independent checks of the energy response, which may also change with time. Furthermore, the density of cometary emission lines is considerably increasing towards energies below $\sim 300 \mathrm{eV}$, so that the spectral reconstruction may not be unique due to spectral blends. While high resolution spectroscopy with slitless X-ray gratings avoids some of these limitations and offers unique possibilities, it poses an additional challenge, because there the spectral response is affected by the spatial extent of the comet along the dispersion direction. In view of all these challenges, it appears that the promising potential of cometary X-rays for charge transfer studies has not yet been fully utilized.

As soon as a good quantitative and detailed understanding of cometary X-ray spectra, consistent with theoretical calculations and laboratory measurements, will have been achieved, it is likely that other research areas using plasma diagnostics will benefit, not only in astrophysical context, but also in applied science, like in the development of nuclear fusion devices (Sect. 3.1). Thus, X-ray observations of comets can be considered as benchmarking experiments for testing our understanding of the physics of charge transfer.

\section{History of Charge Transfer Studies}

For our knowledge of the role of charge transfer in X-ray astrophysics, the year 1996 marks an important dividing point. Before 1996, the physics of charge transfer was studied in various contexts, but one important aspect was completely overlooked: charge transfer can be so efficient that it can be a major or even the by far dominant source of X-ray emission for particular objects. This section will give a brief overview of this exciting development.

\subsection{The Situation Before 1996}

The study of charge transfer reactions has a long history. As these reactions are likely to occur whenever ions encounter neutral atoms, one may argue that charge transfer has accompanied atomic physics from its very beginning, starting with the investigations of the 
scattering of He ions in solids by Geiger and Marsden (1909), which led Rutherford (1911) to deduce the fundamental structure of the atom. Specific studies of the physics of charge transfer were performed already more than 75 years ago, when, e.g., Massey and Smith (1933) investigated the dynamic properties of the passage of ions through gas by a quantum mechanical treatment of the collisions, and when Mulliken (1939) performed a theoretical study of the spectral properties of charge transfer. A very early review about charge exchange studies was presented by Rüchardt (1933).

In the next decades, the investigations continued (for reviews, see Massey and Burhop 1952; Allison and Warshaw 1953) and were also extended to astrophysical scenarios. Perhaps the first of these investigations was devoted to comets: Biermann (1953) suggested that the $\mathrm{CO}^{+}$ions observed in their tails might be predominantly the result of charge transfer reactions between solar wind protons and neutral cometary $\mathrm{CO}$ molecules, and Harwit and Hoyle (1962) concluded that charge transfer may lead to the formation of a magnetic barrier around the cometary nucleus. In the solar system, another study of charge transfer was devoted to the interaction of solar wind protons with neutral particles in the vicinity of the Sun, in order to explain the fast neutral particles entering the Earth atmosphere (Fahr 1968). Wallis (1973) considered strong cooling associated with charge transfer ionizations as one possibility of weakening the shocks ahead of Venus and Mars, and Dalgarno et al. (1980) investigated the effect of charge transfer between $\mathrm{O}^{2+}$ and $\mathrm{He}$ ions on the ion chemistry of the upper atmospheres of these planets as well as on the terrestrial ionosphere.

Charge transfer studies, however, were not restricted to our solar system. In the supernova remnant Cas A, charge transfer with protons was suggested to be responsible for the observed excitation of oxygen in its optical spectrum (Chamberlain 1956), and for the presence of broad iron lines in its X-ray spectrum (Serlemitsos et al. 1973). Watson (1976), Blint et al. (1976), Christensen et al. (1977), and Rule and Omidvar (1979) investigated the effect of charge transfer between low-energy cosmic rays and the interstellar gas for the production of X-ray line emission. Bussard et al. (1978) calculated the X-ray production at $\sim 6.8 \mathrm{keV}$ from Fe ions moving through a medium with solar abundances, following both charge transfer and collisional excitation, and applied it to the galactic center and to the galaxy Cen A. Tarter et al. (1979) studied the influence of double charge transfer processes on the ionization structure and emission spectra of a cosmic gas excited by a variety of ionizing sources. According to Pequignot et al. (1978) and Pequignot (1980b), the problem of explaining the ionization structure of the planetary nebula NGC 7027 by static models based on the standard photoionization theory can be successfully eliminated by including charge transfer reactions with hydrogen. Halpern and Grindlay (1980) considered the effect of charge transfer on the ionization structure of X-ray photoionized nebulae. In their modeling of a bright filament of the Crab Nebula by stationary photonionization, Pequignot and Dennefeld (1983) took also charge transfer reactions into account. By performing ionization equilibrium calculations for interstellar gas at $10^{4}-10^{5} \mathrm{~K}$ subject to diffuse UV and X-ray radiation, Suchkov and Shchekinov (1983) concluded that charge transfer reactions will contribute significantly to the ionization balance, and Wise and Sarazin (1989) investigated the contribution of charge transfer reactions to the X-ray emission of supernova remnants (Sect. 5.1).

From 1973 to 1981, the flybys of the Pioneer and Voyager spaceprobes on Jupiter and Saturn provided unique opportunities for in-situ studies of charge transfer processes (which were usually referred to as 'charge exchange'). During the encounter of Pioneer 10 with Jupiter, it was found that there is a 'plasmasphere' of protons inside the flux tubes of Io with sufficiently high densities to limit the azimuthal extent of the partial torus of hydrogen gas from Io by the mechanism of charge exchange (Frank et al. 1975). In their investigation 
of the heat transport in the Jovian magnetosphere, Machida and Nishida (1978) suggested that hot protons produced at Io's orbit are replaced by cool protons by charge exchange in Io's hydrogen torus. The subsequent Voyager 1 flyby revealed that just outside the orbit of Io, the ion angular distributions are most sharply peaked at $90^{\circ}$ local pitch angle, consistent with diffusion of the particles inward from large radial distances. However, inside the orbit of Io, the angular distributions of lower energy ions were found to be depleted at $90^{\circ}$ local pitch angles, suggesting the possibility of charge exchange scattering loss in the vicinity of the Io torus due to neutral atomic and molecular clouds emitted from Io (Lanzerotti et al. 1981; Ip 1981). Also at Saturn, measurements obtained during the Voyager 1 flyby indicated that charge exchange of energetic ions with satellite tori is an important loss mechanism there (Kirsch et al. 1981). Charge exchange at Jupiter was considered by Horanyi et al. (1988) as one of the basic processes in their theoretical modeling of the energy deposition by precipitating energetic oxygen ions, in order to explain the high UV flux which was observed at the auroral regions with the Voyager 1 and 2 spacecraft and the International Ultraviolet Explorer (IUE) satellite. Cravens et al. (1995) extended this model, which had originally included only the lowest four charge states of oxygen, to all its charge states, and concluded that the auroral X-ray emission of Jupiter observed by the Rosat satellite (Waite et al. 1994) can be explained by heavy ion precipitation.

In addition to the effects listed above, charge exchange has another important consequence: the ions which are neutralized in this process are freed from the electromagnetic field that confines the ion population, and can move to remote regions, providing a sample of the parent ion population to distant observers. Ion populations of different origins produce different Energetic Neutral Atom (ENA) signatures in terms of flux levels, and energy, spatial, and temporal dependence (e.g. Hsieh et al. 1992b). ENAs emitted from the Earth's magnetosphere were detected with the IMP 7/8 and ISEE 1 spacecraft during the recovery phase of two moderate geomagnetic storms in Nov. 1973 and Dec. 1977 at 33 and 20 Earth radii, respectively. They were produced when singly charged trapped ions collided with the neutral hydrogen in Earth's exosphere (Roelof et al. 1985). Hsieh and Curtis (1988) presented a model for the production of ENAs in the Saturn/Titan system and concluded that charge exchange between oxygen and hydrogen in the inner magnetosphere is sufficient to account for all the ENA oxygen production observed by Voyager 1 (Kirsch et al. 1981). There may even be a practical application of ENAs: Hsieh et al. (1992a) suggested to use energetic neutral hydrogen atoms produced during the transit phase of an Earth-directed coronal mass ejection (CME) by charge exchange, which can travel ahead of the CME, to forecast a magnetic storm. This would be possible because CMEs decelerate continuously after their ejection, while the neutral atoms continue to propagate at their initial high speeds.

As the detection of ENAs from different directions provides remotely a global picture of the plasma distribution, it should be possible to utilize the ENAs produced by charge exchange between energetic ions and neutral atoms for the imaging of magnetospheres. This concept, proved at Earth by Roelof (1987), who obtained an ENA image of the storm-time ring current from data taken with ISEE 1 in 1978, opened a promising new experimental technique for investigating the global dynamics of planetary magnetospheres (Keath et al. 1989; Curtis and Hsieh 1989). In the following years, it became apparent that global magnetospheric imaging through the detection of ENAs would be a crucial tool for the advancement of magnetospheric research via remote sensing techniques (Orsini et al. 1994). For Mars, Barabash et al. (1995) presented the basic ideas of how the solar wind protons and accelerated planetary ions can be converted there into ENAs via the charge exchange process, and described a detector which was designed to carry out the first imaging of energetic neutrals from another planet. The idea of using ENAs produced by charge exchange 
between energetic ions and ambient neutral atoms as a diagnostic tool to study planetary magnetospheres from a distance was extended to the investigation of the heliosphere (e.g. Grzedzielski and Rucinski 1990). Here, charge exchange between the different energetic ion populations and the neutral gas in and out of the heliosphere provides a means to investigate the energetic ions of various origins in the heliospheric regions otherwise inaccessible to us (Hsieh et al. 1992b). Based on the Voyager 1/2 energetic ion measurements, Roelof (1992) explored the feasibility of ENA imaging of shock-associated energetic proton populations in the heliosphere. The results indicated that useful heliospheric ENA imaging could be accomplished with the technology which was available at that time. ${ }^{4}$

A very interesting aspect, in view of the later discovery of charge exchange induced Xrays from comets in 1996, is the fact that in the years before, charge exchange at comets was intensively studied: Gombosi et al. (1983) showed that charge exchange between the solar wind and cometary gas, where a fast solar wind ion and a slow cometary neutral are replaced by a fast neutral particle and an slow heavy ion, removes a significant amount of momentum from the solar wind flow and effectively increases the distance of the contact discontinuity (separating the ionosphere of a comet and the solar wind) from the nucleus. Galeev et al. (1985) examined the solar wind flow near comets analytically, taking charge exchange cooling of the mass loaded solar wind into account. In 1986, the possibility to perform in-situ measurements at comet Halley had stimulated further studies of this effect. Shelley et al. (1987) found that charge exchange may be an important solar wind loss process in the coma of comet Halley at $\sim 10^{5} \mathrm{~km}$ distances from the nucleus. Ip (1989) investigated the charge exchange effect in the vicinity of the cometopause (separating the solar wind controlled external and the heavy cometary ion dominated inner regions) of comet Halley in order to explain the densities measured with the Vega and Giotto spaceprobes during their encounters with this comet, and Fuselier et al. (1991) reported that the Giotto observations of solar wind ions show charge exchange effects and solar wind compositional changes in the coma of comet Halley.

Parallel to the astrophysical investigations of charge exchange, this process was also investigated in the context of developing an X-ray laser (Scully et al. 1974; Louisell et al. 1975; Krasilnikov 1975), because a significant population inversion can be obtained by resonance charge transfer pumping (Elton et al. 1978). This approach has the advantage that, by picking up an electron in the outer shell of a H-, He-, or Li-like ion, the rapid Auger rates which are characteristic of many other X-ray laser schemes can be avoided (Cantrell et al. 1974). It is also interesting to note that Presniakov and Shevelko (1982) reported the presence of intense X-ray emission when the burst of a hot laser plasma reaches a solid surface, and attributed this effect to charge exchange of multiply charged ions with neutral atoms and low charge ions near the solid surface.

In the late 1970s it was found that charge transfer reactions can be utilized as a valuable diagnostic tool in nuclear fusion, because charge exchange between neutral $\mathrm{H}$ and ionized impurities provides a unique method for measuring the concentrations of impurity nuclei. Since that time, the use of charge exchange spectroscopy (CXS) as a diagnostic technique in high temperature (particularly tokamak) plasmas has become widespread (Carolan et al. 1987). CXS provides fundamental parameters for characterizing fusion plasmas, unparalleled by any other diagnostic technique in detail, accuracy, reliability, and experimental ease

\footnotetext{
${ }^{4}$ Currently this observational technique is being successfully applied: the ENA imagers on the CassiniHuygens mission to Saturn (Krimigis et al. 2009) and on the International Boundary Explorer (IBEX; McComas et al. 2009; Fuselier et al. 2009; Funsten et al. 2009; Schwadron et al. 2009; Möbius et al. 2009) have revealed an unexpected, bright, narrow ribbon of ENA emission from the outer heliosphere, which was not predicted by any model or theory.
} 
(Isler 1994). Charge exchange is also the dominant process in tokamak fusion devices during neutral beam heating and in the edge regions of magnetic fusion plasmas (Boileau et al. 1989; Isler 1994; Beiersdorfer et al. 1999), and the associated energy loss may be the key for the success of radiative divertors in future reactor-type devices (Beiersdorfer et al. 2000). There was also a connection to astrophysics, as the observations of X-ray emission due to charge exchange in laboratory fusion devices (e.g. Rice et al. 1986) motivated Wise and Sarazin (1989) to perform detailed calculations about the possible occurrence of CXE in supernova remnants, but they found its contribution to the overall X-ray emission not to be significant (Sect. 5.1).

As this short and not necessarily complete overview illustrates, there has been a long history and a wide variety of detailed studies of the charge transfer process, by theoretical and experimental investigations and applications. A major fraction of these studies was devoted to astrophysical scenarios, in particular in the solar system. While the importance of charge transfer reactions for calculating line emissivities and determining the ionization balance of astrophysical plasmas was recognized (Field and Steigman 1971; Williams 1973; Baliunas and Butler 1980; Pequignot 1980a; Dalgarno and Sternberg 1982; Shields et al. 1983; Allan et al. 1988) as well as their thermal effects (Kallman and McCray 1982), the perhaps most important aspect of charge transfer reactions for astrophysics, namely their high efficiency for generating 'standalone' $\mathrm{X}$-rays under appropriate conditions, was overlooked.

\subsection{Charge Transfer Reactions in the Rosat Era and Beyond}

The discovery of the importance of charge transfer reactions for X-ray astrophysics is closely related to a particular satellite: Rosat. This link is not coincidental. Our Sun is a source of highly charged ions, which originate in the hot solar corona and are ejected in the solar wind with velocities of $\sim 400 \mathrm{~km} \mathrm{~s}^{-1}$ or more. Due to the rapidly expanding solar wind plasma, they have no possibility to recombine, and remain in their highly charged state until they encounter gas, as it is present in, e.g., the atmosphere of the Earth and comets. Then, recombination by charge transfer becomes possible, and X-rays may be emitted. Due to the high cross section of charge transfer, these are extended sources, which may even span the whole sky, as the Earth exosphere, when viewed from inside. The fact that the $\sim 10^{6} \mathrm{~K}$ temperature in the solar corona is frozen in the charge states of the heavy ions, implies that the emission following charge transfer occurs mainly at energies below $1 \mathrm{keV}$. Thus, the ideal satellite to discover solar wind charge exchange (SWCX) induced X-rays would be one which is sensitive to low energy $\mathrm{X}$-rays, provides a large field of view at a very low instrumental background, and would preferentially scan the whole sky.

The key mission design of Rosat (Trümper 1982) was to perform the first ever all-sky $\mathrm{X}$-ray survey with an imaging telescope. For this purpose, the X-ray telescope was optimized for high sensitivity and a large field of view ( $2^{\circ}$ diameter). These two design goals, together with the requirements of high reflectivity of the X-ray mirrors at grazing incidence angles, restricted the spectral bandpass to energies less than $\sim 2 \mathrm{keV}$. The detector, a position sensitive proportional gas counter (PSPC; Pfeffermann and Briel 1983), was equipped with a highly efficient five sided anticoincidence system (Metzner 1986) to suppress the particle induced background to less than 1 count per day per $\operatorname{arcmin}^{2}$ (Plucinsky et al. 1993). During its all-sky survey, the satellite was continuously scanning the sky along great circles, providing an essentially unlimited field of view. Although charge exchange was not considered in the design of the Rosat mission, it appears that, in retrospect, this mission was specifically tailored to revealing this yet undiscovered fundamental process for the generation of celestial X-rays. 

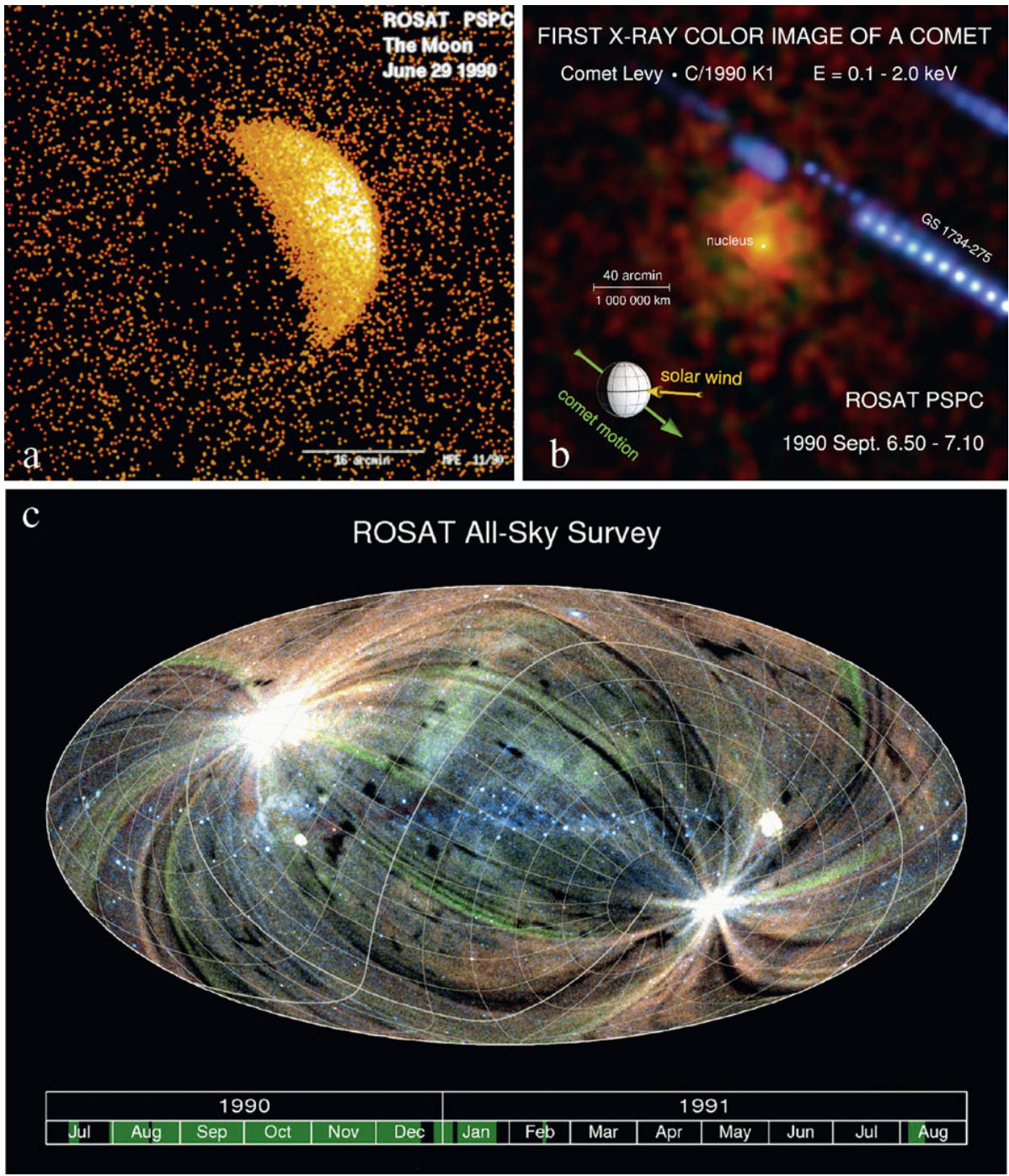

Fig. 1 Evidence for charge exchange induced X-ray emission recorded in the first months after the launch of Rosat: a X-rays from the geocorona which appear to come from the direction of the dark Moon (reprinted with permission from Schmitt et al. 1991), b X-rays from comets, e.g., Comet C/1990 K1 (Levy), one of four comets which were serendipitously observed during the Rosat all-sky survey, cenhanced X-ray flux observed during specific scans in the Rosat all-sky survey

In fact, already on 29 June 1990, only 28 days after launch, when Rosat was still in its performance verification phase, the Moon was observed (Schmitt et al. 1991). The image exhibited X-rays which appeared to originate from the dark side of the Moon (Fig. 1a), a phenomenon which was not understood at that time. Soon afterwards, on 31 July 1990, just one day after the regular Rosat all-sky survey had started, the first comet (45P/HondaMrkos-Pajdušáková) happened to get into the field of view (Dennerl et al. 1997). Its faint $\mathrm{X}$-ray signal, however, was well hidden among the $\sim 100000 \mathrm{X}$-ray sources which Rosat detected in its survey (Voges et al. 2000). Four other comets, serendipitously observed in a 
total of six occasions during the survey (e.g. Fig. 1b), shared the same fate (Dennerl et al. 1997). During the survey phase, however, another phenomenon was observed: 'a mysterious X-ray background that varies on a timescale of $\sim 1-2$ days' (Snowden et al. 1994). These 'long term enhancements' clearly show up in the uncorrected Rosat all-sky image as brightenings along the scan direction (Fig. 1c).

Today we know that all these effects are caused by the same process: charge transfer reactions between highly charged heavy solar wind ions and neutral gas around the Earth or a comet. Finding this explanation was triggered by a rare celestial event: a very close encounter of the Earth with a comet. On 25 March 1996, comet C/1996 B2 (Hyakutake) passed the Earth at a distance of only 0.10 astronomical units. It was mainly the unique opportunity to search for unusual and low luminosity phenomena which motivated an observation of this comet with Rosat. There were some vague ideas how X-rays might be generated, but these did not include charge transfer reactions. Even after the discovery that this comet was a bright X-ray source, when various possible scenarios were investigated, charge transfer reactions were not among them (Lisse et al. 1996b). Despite the difficulties in finding a satisfactory explanation, the subsequent detection of X-ray emission from comets in archival Rosat data (Dennerl et al. 1996a, 1996b, 1996c) and additional observations of C/1996 B2 (Hyakutake; Lisse et al. 1996c) and C/1996 Q1 (Tabur; Dennerl et al. 1996d) clearly showed that X-ray emission is a class property of comets. It also indicated that the emission is related to their gas rather than their dust content (Dennerl et al. 1997). The fact that there was obviously a mechanism capable of causing the cold cometary gas to emit X-rays suggested that, independent of the missing explanation, a similar effect might also operate in the Earth's exosphere, causing it to glow in X-rays (Dennerl et al. 1997; Cox 1998; Freyberg 1998). This would immediately explain the 'long term enhancements' observed in the Rosat all-sky survey and the X-rays which appeared to come from the direction of the dark Moon.

The mystery of cometary X-ray emission was solved by Cravens (1997), who suggested that the emission is caused by charge transfer of solar wind heavy ions and cometary gas. His model combined known properties of comets, of the solar wind, and of atomic physics, to make detailed predictions about the observable X-ray morphology, luminosity, and spectrum as well as a general prediction of the temporal variability. These predictions were found to agree with the Rosat data of all the comets which were observed in the all-sky survey (Dennerl et al. 1997) and of comet Hyakutake (Wegmann et al. 1998), as well as with data obtained with EUVE (Krasnopolsky 1997; Krasnopolsky et al. 1997; Mumma et al. 1997; Krasnopolsky and Mumma 2001). Also the further X-ray and Extreme Ultraviolet (EUV) observations of comets, obtained with Rosat (Lisse et al. 1999) and EUVE (Krasnopolsky et al. 2000), were found to be consistent with solar wind charge exchange (SWCX). ${ }^{5}$ In particular, detailed comparisons between the morphology observed with Rosat and XMMNewton and that expected for charge exchange yielded excellent agreement (Wegmann et al. 2004), and by utilizing the high sensitivity of XMM-Newton, it was even possible to deduce the location of the cometary bow shock (Wegmann and Dennerl 2005). The improvement in spectral resolution, which became available, just at the right time, with X-ray CCDs onboard the satellites Chandra and XMM-Newton, directly revealed the characteristic X-ray emission lines expected from charge transfer reactions (Lisse et al. 2001; Krasnopolsky et al. 2002;

\footnotetext{
${ }^{5}$ An X-ray observation of Comet C/1995 O1 (Hale-Bopp) with BeppoSAX was interpreted by scattered solar X-rays on very small dust grains (Owens et al. 1998, 1999a, 1999b), but CXE could not be observationally ruled out (Schulz et al. 2000), and Kharchenko and Dalgarno (2001) have interpreted the X-ray spectrum in terms of CXE characteristic of a slow solar wind.
} 
Dennerl et al. 2003; Kharchenko et al. 2003), which were resolvable before only in the EUV range (Krasnopolsky and Mumma 2001). The X-ray spectrum with the highest resolution currently available, obtained from comet C/2000 WM1 (LINEAR) with the Reflection Grating Spectrometers onboard XMM-Newton (RGS; den Herder et al. 2001), even allows the multiplet of He-like O VII emission to be resolved and to prove unambiguously the presence of charge transfer reactions (Dennerl et al. 2011). In short, comets can now be considered as the best natural laboratory for studying charge transfer reactions. They have demonstrated the efficiency of these reactions for creating X-rays and have directed our attention to the fact that nature is capable of producing extremely favorable conditions for the generation of CXE. This, however, was overlooked for a long time and only discovered by chance.

In retrospect, with the facts presented above in mind, it may seem surprising that the discovery of cometary X-ray emission in 1996 was considered as such a big surprise, because all the ingredients necessary to predict this emission were already available since 1968: the properties of the cometary coma (Haser 1957), the solar wind velocity (Biermann 1951) and its heavy ion content (Bame et al. 1968; Hundhausen et al. 1968), the average cross sections of charge transfer reactions (Bohr and Lindhard 1954), and the energies released in the subsequent recombination (e.g. Tucker and Gould 1966). All what would have been needed in order to get an estimate of the X-ray luminosity $L_{\mathrm{x}}$ of a comet was to multiply the following numbers: ${ }^{6}$

$$
L_{\mathrm{x}} \sim v_{\mathrm{sw}} n_{\mathrm{sw}} y_{\mathrm{x}} E_{\mathrm{x}} S_{\mathrm{c}}
$$

i.e., the solar wind velocity $v_{\mathrm{sw}} \sim 400 \mathrm{~km} \mathrm{~s}^{-1}$, the solar wind particle density at the location of a comet $n_{\mathrm{sw}} \sim 10 \mathrm{~cm}^{-3}$, the fraction of heavy ions in the solar wind capable of releasing an X-ray photon in a charge exchange event $y_{\mathrm{x}} \sim 10^{-3}$, the average X-ray energy $E_{\mathrm{x}} \sim$ $0.3 \mathrm{keV}$ released per charge exchange event, and the projected area of the cometary coma $S_{\mathrm{c}}=r_{\mathrm{c}}^{2} \pi \sim 5 \cdot 10^{12} \mathrm{~km}^{2}$. With these values, an impressive X-ray luminosity of $\sim 10^{9} \mathrm{~W}$ results, corresponding to an easily observable X-ray flux of $\sim 1.6$ photons s$~^{-1}$ from $10^{8} \mathrm{~km}$ distance. Thus, the prediction that comets constitute a new class of X-ray sources would have been possible already in 1968.

The lack of such a prediction is even more surprising if one considers that, on the one hand, there were ideas around how the emission generated by charge transfer could be utilized in the laboratory, and that, on the other hand, charge transfer was intensively studied for decades in astrophysics, in particular at comets, culminating with in-situ measurements at comet Halley, where the Giotto data showed the charge of solar wind oxygen and carbon ions to decrease with decreasing distance to the cometary nucleus, a clear indication for the presence of charge transfer (Fuselier et al. 1991).

The serendipitous aspect in the discovery of the importance of charge transfer for X-ray astrophysics, by revealing the X-ray emission of comets, has a famous counterpart in X-ray astronomy: the serendipitous discovery of X-rays from an accreting neutron star, Sco X-1, in June 1962 (Giacconi et al. 1962). This discovery, which marks the birth of X-ray astronomy, was also considered as a big surprise. However, with the information which was available at that time, i.e., the concept of neutron stars (Landau 1932; Baade and Zwicky 1934) and of Roche lobe overflow in close binaries with subsequent accretion (Crawford and Kraft 1956),

\footnotetext{
${ }^{6}$ This estimate is based on the fact that, due to the high cross section of charge transfer, essentially each heavy ion in the solar wind is subject to at least one charge transfer process in the coma within its assumed extent, and that the released X-rays can leave the coma unattenuated, because the cross section for interaction of $\mathrm{X}$-ray photons is four orders of magnitude lower than that of heavy ions.
} 


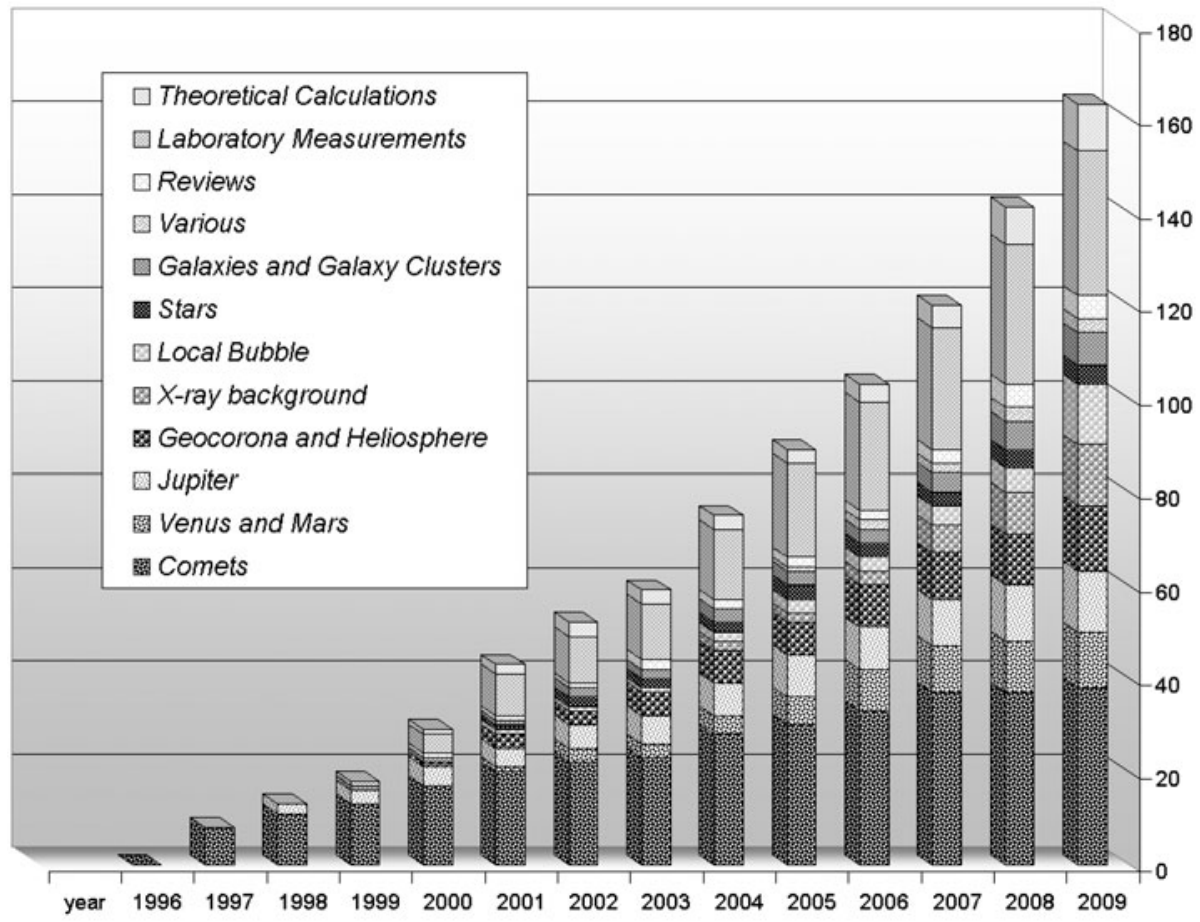

Fig. 2 Cumulative number of refereed publications on charge transfer reactions which are related to the discovery of cometary X-ray emission (except for some publications on Jupiter, which describe independent charge transfer studies). The publications are grouped according to the categories listed in the legend. Publications which cover several topics are assigned to their main category

it would have been a straightforward exercise ${ }^{7}$ to predict the dominance of accreting neutron stars in the X-ray sky. However, in 1962, it was expected that lunar fluorescence from solar $\mathrm{X}$-rays might be the strongest source in the night sky (Giacconi 2003).

After the discovery of CXE in comets, the importance of charge transfer reactions for generating $\mathrm{X}$-rays in astrophysical environments became obvious and has stimulated many further investigations of CXE, including new dedicated laboratory experiments and theoretical calculations as well as astrophysical studies. The expansion of this field of research is clearly visible in Fig. 2, where the cumulative number of refereed publications on charge transfer reactions since 1996 is displayed. With the exception of some publications on Jupiter, where charge transfer was independently investigated, only those publications were included which are explicitly related to the discovery of cometary X-ray emission. Figure 2 illustrates how this discovery has stimulated the interest of the astrophysical community in

${ }^{7}$ Matter falling onto a neutron star with a mass $M=1 \mathrm{M}_{\text {sun }}$ and a radius $R=10 \mathrm{~km}$ releases $G M R^{-1} c^{-2}=$ $15 \%$ of its rest mass as thermal energy. By balancing the gravitational pressure and the radiation pressure, it follows that, for spherical inflow, an accretion rate of $\sim 1.5 \cdot 10^{-8} \mathrm{M}_{\text {sun }} \mathrm{yr}^{-1}$ is already sufficient to saturate the accretion process. From the corresponding Eddington luminosity, $L_{\text {Edd }} \sim 1.3 \cdot 10^{38} \mathrm{erg} \mathrm{s}^{-1}$, the temperature $T$ of the emitting plasma can be estimated by $L_{\mathrm{Edd}}=4 \pi R^{2} \sigma T^{4}$. The resulting high temperature, $T \sim 2 \cdot 10^{7} \mathrm{~K}$, implies that the energy is mainly radiated in the X-ray range. The X-ray luminosity is so high that even from a distance of $1 \mathrm{kpc}$, a flux of $10^{-6} \mathrm{erg} \mathrm{cm}^{-2} \mathrm{~s}^{-1}$ is observed. This is four orders of magnitude higher than the X-ray flux which we get from the Moon. 
the CXE mechanism: while papers on comets dominate the publications on CXE in the first years after 1996, this field has expanded so much that, by 2009, the publications on comets comprise less than one quarter. This is a clear indication that the consequences of this discovery go far beyond cometary research. The following two Sections will attempt to present a concise overview on our current knowledge about the various locations where CXE is known or likely to occur and has or may have been detected.

\section{Solar System X-rays due to Charge Transfer Reactions}

The fact that the Sun is emitting highly charged heavy ions which are producing CXE whenever they encounter gas, and that gas is found at various places in the solar system, makes our solar system rich in CXE sources, as will be described in this section.

\subsection{Comets}

Comets are the most prominent CXE sources. Their importance for fundamental studies of the charge transfer process was already discussed in Sect. 2.3, and in Sect. 3.2 a short summary of some scientific highlights on cometary X-ray emission was presented. Here, a novel possibility of utilizing the X-ray emission of comets is briefly addressed: remote sensing of the heavy ion content of the solar wind.

After it was realized that cometary X-ray emission is the result of charge transfer interactions between heavy solar wind ions and neutral gas, it became obvious that comets can be used as natural probes for monitoring the heavy ion content of the solar wind (Dennerl et al. 1997; Kharchenko and Dalgarno 2000), because each ion leaves its characteristic signature in the X-ray spectrum. Around solar minimum, two types of solar wind are present: a fast $\left(v \sim 700 \mathrm{~km} \mathrm{~s}^{-1}\right)$, steady polar component at latitudes above $\sim 20$ degrees, characterized by low density and low ionization, and an equatorial component, which is typically slow $\left(v \sim 400 \mathrm{~km} \mathrm{~s}^{-1}\right)$, dense, and highly ionized, but also highly variable in these parameters. Outside solar minimum, the equatorial component is expanding to higher latitudes, so that the clear distinction between both components disappears around solar maximum. Before the discovery of cometary X-ray emission, an investigation of the chemical composition and ionization state of the solar wind required in-situ measurements, and to date only one instrument, SWOOPS on Ulysses (Bame et al. 1992), was able to perform such measurements at high heliographic latitudes (McComas et al. 2003).

In this context, the cometary X-ray emission holds a high scientific potential. The large extent of the cometary coma combined with the large cross section for charge transfer reactions makes comets act as sensitive sensors (cf. Fig. 4b) which probe locally the heavy ion content of the solar wind and transmit this information by electromagnetic radiation (X-rays) over large distances. Moreover, due to the fact that, unlike all other solar system objects, the paths of comets are not restricted to low ecliptic latitudes, they allow a full 3D sampling of the solar wind. As the Oort cloud and the Kuiper belt provide, on the long-term average, a constant supply of comets, the solar wind can be monitored over all phases of the 11 year solar activity cycle. Thus, comets are ideally suited for deriving observational information about fundamental properties of the solar wind, which would be very difficult to obtain otherwise. This information is not only important for our understanding of the Sun, but also of solar-type stars in general.

The first quantitative attempts to deduce solar wind properties from cometary Xrays were made by Schwadron and Cravens (2000), using archival Rosat data of comet 
C/1990 K1 (Levy), the brightest comet which was observed in the all-sky survey, ${ }^{8}$ and by Kharchenko and Dalgarno (2001), who also included the EUVE spectrum of comet Encke and the BeppoSAX spectrum of comet Hale-Bopp. These two investigations, however, arrived at different conclusions about the solar wind properties. This discrepancy is probably not only related to different assumptions, but also to the limited spectral resolution which was available in these early data. In the year 2000 the situation improved considerably with the launch of Chandra and XMM-Newton, which are both equipped with X-ray CCDs. Over the last decade, a sufficient number of comets was observed with these satellites to allow for improved studies of whether they can indeed be utilized as remote sensors of the heavy ion content of the solar wind. Krasnopolsky (2006) analyzed the X-ray spectra of four comets which were observed with Chandra in a consistent way and found significant variability of the flow, chemical composition, and charge state of the solar wind.

Bodewits et al. (2007) analyzed the X-ray spectra of all the comets, eight in total, which were observed with Chandra in the period 2000 to 2006, covering the transition from solar maximum to solar minimum. The analysis was done in a consistent way for all the comets, by keeping the relative intensities of all transitions of each ion (i.e., C V, C VI, N VI, N VII, $\mathrm{O}$ VII, O VIII) fixed according to emission cross sections which were independently determined for several collision velocities, and by adding two emission lines of $\mathrm{Ne}$ IX and $\mathrm{Ne} X$. In this way, the number of free parameters could be reduced from 35 to 8 , making the spectral fits tractable. Figure 3a shows the (background subtracted raw) spectra of all the comets, and Figs. 3b, c show at which ecliptic latitude and phase in the solar cycle the spectra were observed. It is immediately obvious that there are spectral differences. In Fig. 3a, three spectral bands are indicated, dominated by emission from (i) C V, C VI, N VI ('C + N'), (ii) by $\mathrm{O}$ VII, and (iii) by O VIII ions, and the spectra are arranged in the way that, from top to bottom, flux is systematically shifted from lower to higher energy bands. The quantitative results of the spectral fits clearly show that the flux in the $\mathrm{C}+\mathrm{N}$ band is anticorrelated to that in the O VIII band (Fig. 3d), indicating that the comets were exposed to different solar wind conditions.

As can be seen in Fig. $3 b$ and c, all the comets which were observed at high latitudes happened to be there during solar maximum, when the equatorial solar wind had expanded into these regions. This implies that, until 2006, Chandra had not observed any comet exposed to the polar wind. This situation changed in October 2007, during solar minimum, when comet 17P/Holmes experienced a spectacular outburst, which increased its optical brightness within hours by almost a million times, from under 17 mag to $3 \mathrm{mag}$, making it the optically by far brightest comet which was observable with Chandra since its launch. Comet 17P/Holmes was placed at a sufficiently high latitude $\left(19^{\circ}\right)$ to be exposed to the polar wind. It was thus expected that this comet would exhibit considerably different X-ray properties, and in fact this was observed: 17P/Holmes became the first comet where Chandra did not detect any significant X-ray emission at all (Christian et al. 2010). The most likely explanation for this dramatic X-ray faintness is that the polar wind was so diluted and its ionization so low that only very little CXE was generated at energies above $\sim 300 \mathrm{eV}$. An instrumental effect, i.e., a loss of sensitivity, can definitively be ruled out, because only two months later, another comet, 8P/Tuttle, was observed with Chandra, and this comet, at low latitude $\left(3^{\circ}\right)$, was clearly detected in X-rays (Christian et al. 2010).

\footnotetext{
${ }^{8} \mathrm{~A}$ spectral analysis of the Rosat data of comet Hyakutake was not possible, because this comet was observed with an X-ray detector ('HRI') of insufficient energy resolution. The comets which were found in the data of the Rosat all-sky survey, however, were observed with a proportional counter ('PSPC') which provided some spectral information.
} 

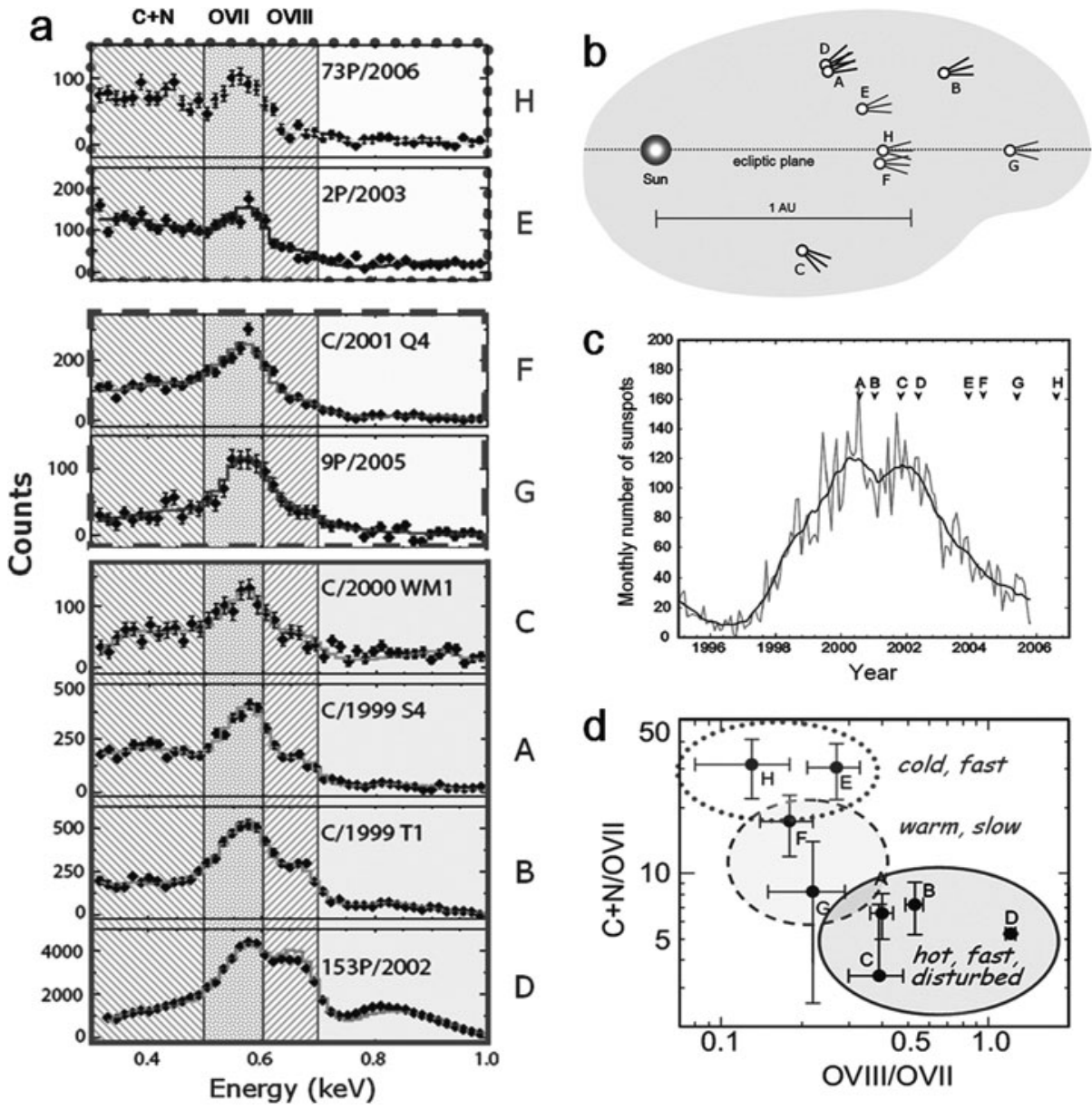

Fig. 3 Summary of the spectral results obtained with Chandra for all the comets (denoted by A-H) which were observed from 2000 to 2006: a the $0.3-1.0 \mathrm{keV}$ pulse height distributions, b the ecliptic latitudes and c phases in the solar cycle of the observed comets, and $\mathbf{d}$ the deduced information about the solar wind heavy ion content. All the comets were exposed to the equatorial solar wind, because of their low ecliptic latitude $(\mathrm{E}-\mathrm{H})$ or the fact that they were observed during solar maximum (A-D), when the equatorial solar wind extends to high latitudes. Three spectral bands are shown in (a), which are dominated by emission from $\mathrm{C}+\mathrm{N}$, O VII, and O VIII, respectively; the spectra are arranged in the way that they become progressively harder from top to bottom. Consequently, the flux ratios in the three bands derived by spectral fits (d) fall into three categories, indicative of cold, fast (top), warm, slow (middle), and hot, fast, disturbed (bottom) solar wind conditions. a, c, d were adapted from (Bodewits et al. 2007)

These observations have demonstrated how sensitively the cometary X-ray emission responds to changes in the solar wind, and that comets can indeed be used for remote sensing of the heavy ion flux. However, they also show that the spectral resolution which can be obtained from the X-ray CCDs onboard Chandra and XMM-Newton is a limiting factor below $E \sim 300 \mathrm{eV}$, where the density of emission lines becomes so high that a unique reconstruction of the incident photon spectrum is not possible without model assumptions. Higher spectral resolution will be required in order to fully exploit the scientific potential contained in cometary X-rays. 


\subsection{Nonmagnetic Planets with Atmospheres}

At nonmagnetic planets possessing atmospheres, i.e., Venus and Mars, the solar wind interacts in a similar way with their outer atmospheres (exospheres) as it interacts with comets. In this respect, Venus and Mars can be considered as planetary analogs to comets. Compared to the coma of a comet, however, there are marked differences (e.g. Dennerl 2009): the atmosphere of a planet is much more condensed, which implies that only the outermost atmospheric regions are capable of emitting X-rays by charge exchange, because the solar wind heavy ions are exhausted when they reach the inner atmospheric layers. Thus, the charge exchange induced surface X-ray brightness of a planetary atmosphere does not exceed that of the dense inner part of a collisionally thick cometary coma. As the atmospheres of Venus and Mars subtend a considerably smaller solid angle than comets, when observed from near Earth, their CXE flux is easily exceeded by that of a comet.

The high density of a planetary atmosphere has another consequence: it causes a competing process for the production of X-rays to become important: scattering of solar X-rays. As the cross sections for scattering $\left(<10^{-18} \mathrm{~cm}^{2}\right)$ are at least three orders of magnitude smaller than those for charge exchange $\left(\sim 10^{-15} \mathrm{~cm}^{2}\right)$, scattering requires a sufficient density of the target gas in order to obtain a sufficient optical depth for photoabsorption and subsequent reemission. This is the case in a planetary atmosphere (at a height of $110 \mathrm{~km}$, e.g., the atmospheric density is $\sim 10^{12} \mathrm{~cm}^{-3}$ at Mars, and $\sim 10^{14} \mathrm{~cm}^{-3}$ at Venus), but not in a cometary coma (even for an active comet with a gas production rate of $10^{29}$ molecules s $\mathrm{s}^{-1}$, the density at $1000 \mathrm{~km}$ distance from the nucleus is only $\sim 10^{7} \mathrm{~cm}^{-3}$ ). Depending on the relative strengths of the solar X-ray and heavy ion flux, scattering of solar X-rays may even dominate the overall X-ray flux. This was the case in the first X-ray observations of Venus (Dennerl et al. 2002) and Mars (Dennerl 2002). These observations have shown that the scattering occurs predominantly in the form of fluorescence on $\mathrm{C}, \mathrm{O}$, and $\mathrm{N}$ atoms (the main atmospheric constituents), where an X-ray photon is emitted after removal of an innershell electron by photoionization. The atomic transitions which give rise to the emission of an $\mathrm{X}$-ray photon are similar to those in the deexcitation process after charge exchange. However, in the case of CXE, the atom is in a higher ionization state, which implies that there are less electrons present to shield the positive nuclear charge, thus increasing the binding energy of the inner shells. Consequently, the X-ray energies resulting from CXE are somewhat (by a few percent) higher than the corresponding transitions from fluorescence. This offers a possibility to spectrally distinguish between both processes. With the high spectral resolution which is provided by $\mathrm{X}$-ray gratings, it is even possible to resolve fine structure in the fluorescent emission, resulting from molecular transitions in $\mathrm{CO}_{2}$, as demonstrated on Venus with Chandra (Dennerl et al. 2002) and on Mars with XMM-Newton (Dennerl et al. 2006).

The additional presence of scattered solar X-rays (Cravens and Maurellis 2001) poses a major challenge for observing CXE (Holmström et al. 2001, 2002) at nonmagnetic planets. A separation between components can be achieved, within limitations, by utilizing the spectral, spatial, and temporal information available. For Mars, which possesses a very extended exosphere due to its low mass (only $10.7 \%$ of the Earth), the spatial separation is fairly straightforward. Indications for CXE from an extended X-ray halo were found already in the first Chandra observation, though at low statistical significance (Dennerl 2002). The existence of the halo was confirmed by a subsequent XMM-Newton observation with higher sensitivity (Dennerl et al. 2006), which made it even possible to image the extended emission in the light of individual X-ray line transitions (Fig. 4a). For Venus, being 7.6 times more massive than Mars, the exosphere is considerably more compact, which makes a spatial discrimination between $\mathrm{CXE}$ and scattered solar X-rays a very challenging goal. This 

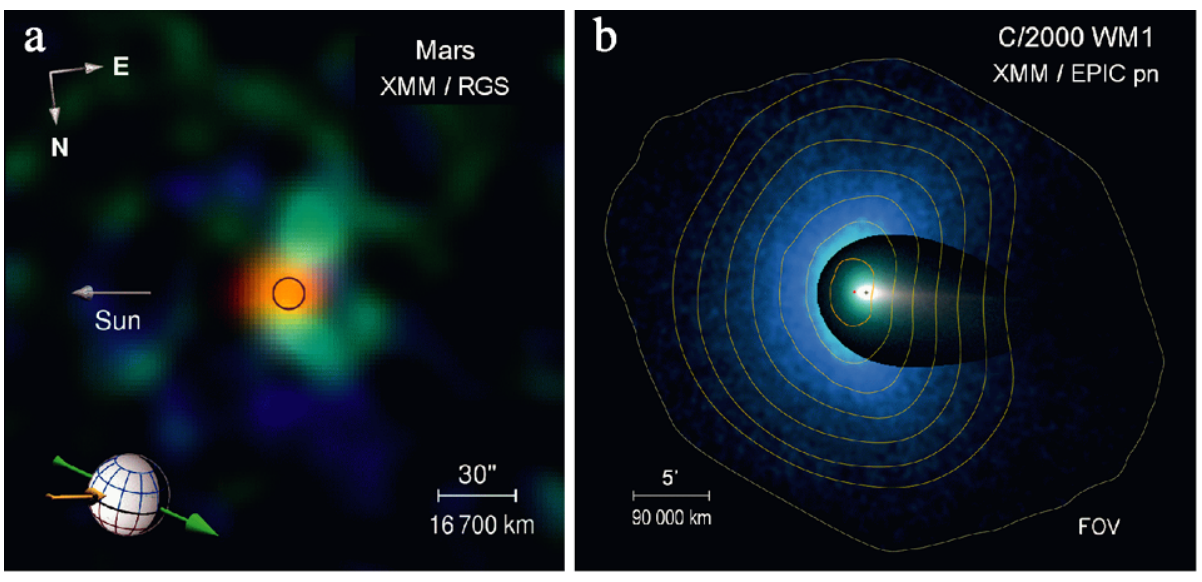

Fig. 4 Demonstration of the capability of charge exchange induced X-ray emission of revealing tenuous amounts of gas. a XMM-Newton/RGS image of Mars, showing emission from ionized solar wind oxygen and carbon in blue and green, respectively, while fluorescence of solar X-rays on neutral carbon and oxygen is coded in orange (Dennerl et al. 2006). b XMM-Newton/EPIC pn image of Comet C/2000 WM1 with contour lines, superimposed on an optical image (Dennerl et al. 2003). In both images, the Sun is to the left

goal can be attained by utilizing the following two circumstances: (i) Simulations indicate that CXE should be almost exclusively confined to the limb (Gunell et al. 2007), while the limb brightening expected from scattered solar X-rays should be less pronounced (Dennerl et al. 2002). (ii) The solar X-ray luminosity, and thus the solar X-ray flux scattered off the Venusian atmosphere, is considerably higher modulated with the $\sim 11$ year solar cycle than the equatorial solar wind. As the X-ray image of Venus is a superposition of a scattered solar $\mathrm{X}$-ray image with moderate limb brightening and a CXE image with high limb brightening, the overall limb brightening should increase from solar maximum to solar minimum. Chandra observations from 2001 (solar maximum) to 2007 indeed show this behaviour, as well as indications for a spectral transition from primarily scattered solar X-rays to CXE from the center to the limb (Dennerl 2008). Thus, for Venus and Mars, the presence of CXE has been observationally established.

$\mathrm{X}$-ray observations of charge exchange interactions between atmospheric constituents and solar wind ions in planetary exospheres have a high scientific potential, because this process is considered as an important nonthermal escape mechanism, which may be responsible for a significant loss of the atmosphere. Although this escape process is mainly due to charge exchange with solar wind protons, which are $\sim 1000$ times more abundant than heavy ions and which do not produce X-rays, the high cross section makes CXE an efficient tracer of planetary outgassing (e.g. Fig. 4a). X-ray observations open up a completely new possibility of remote, global imaging of planetary exospheres, and their spatial and temporal variability (Dennerl 2006).

\subsection{Magnetic Planets with Atmospheres}

Charge exchange occurs also in the atmospheres of magnetic planets, and in fact this is where hints to this process had first been found (Sect. 3.1). Compared to nonmagnetic planets, the presence of a strong magnetic field deflects and accelerates the heavy ions and modifies the charge exchange mechanism considerably. When ions precipitate at high kinetic 
energies into a planetary atmosphere, collisions with atmospheric atoms and molecules reduce the ion translational energies and increase the fraction of highly charged ions. Thus, in contrast to nonmagnetic environments, the charge of the precipitating ions may increase and decrease many times in sequential collisions with the atmospheric gas. In this case, the $\mathrm{X}$-ray spectra depend strongly on the kinetic ion energies, while in the nonmagnetic case, they are determined by the composition of the solar wind. This was investigated in detail by Kharchenko et al. (2008) for Jupiter.

Jupiter is the planet with the highest magnetic field in the solar system, and a powerful $\mathrm{X}$-ray source. X-rays from Jupiter, coming from both polar regions, had already been detected in observations with the Einstein satellite in 1979 and 1981 (Metzger et al. 1983). Further observations with Rosat in 1994 revealed additional X-ray emission at low latitudes (Waite et al. 1997). A detailed interpretation, however, was hampered by the limited spatial and spectral resolution available. Substantial progress in the interpretation was obtained with the satellites Chandra (e.g. Gladstone et al. 2002; Elsner et al. 2005a; Bhardwaj et al. 2006) ${ }^{9}$ and XMM-Newton (e.g. Branduardi-Raymont et al. 2004, 2006, 2007b, 2008; Bhardwaj et al. 2005b). They showed that the equatorial component is predominantly caused by scattered solar X-rays (Maurellis et al. 2000; Bhardwaj et al. 2005b; Cravens et al. 2006; Branduardi-Raymont et al. 2007b), similar to Venus and Mars, and indicated that charge exchange is the basic explanation of the polar component for X-ray energies below $\sim 2 \mathrm{keV}$, while bremsstrahlung of energetic electrons precipitating from the magnetosphere may be responsible for the X-ray emission at higher energies (Branduardi-Raymont et al. 2004, 2006, 2007a, 2008). In contrast to the nonmagnetic planets, however, where the heavy ions are supplied by the solar wind, the heavy ions could also originate from the Jovian magnetosphere, by acceleration and subsequent additional ionization of ambient sulfur and oxygen ions in a field-aligned potential (Bunce et al. 2004), as explored by Cravens et al. (2003), who considered the second possibility more likely. Also Hui et al. (2009) favored the idea that ions of magnetospheric origin dominate in driving the auroral X-ray emission, because fits to the X-ray spectra of the Jovian aurora obtained with Chandra showed no improvement by including carbon, which would be abundant in the solar wind.

Saturn also possesses a high magnetic field, rotates fast, exhibits powerful UV aurorae, and has been detected in X-rays with XMM-Newton and Chandra (Ness et al. 2004a, 2004b). Thus, by analogy with Jupiter, one might expect that auroral X-ray emission should be observable at Saturn, too. None, however, has been detected to date. Almost all of the observed X-ray flux from Saturn is caused by scattered solar X-rays (Bhardwaj et al. 2005a; Cravens et al. 2006), with the possible exception of X-rays from its rings (Bhardwaj et al. 2005c), where other processes may also contribute, like lightning induced electron beams, which might energise the icy particles in the rings and cause them to emit fluorescent $\mathrm{O}-\mathrm{K}_{\alpha}$ radiation (Branduardi-Raymont et al. 2010). The lack of any observable auroral X-ray emission from Saturn is consistent with its low FUV emission compared to Jupiter, and by its at least $\sim 20$ times lower surface magnetic field, magnetic moment, and magnetic particle density, which makes it likely that any auroral X-ray emission is below the sensitivity limit of current instrumentation, as pointed out by Branduardi-Raymont et al. (2010). The same authors estimated that the expected auroral X-ray flux from Uranus and Neptune is also too faint to be currently detected (for Uranus, this estimate is in agreement with a non-detection by Chandra in August 2002; Bhardwaj et al. 2007), and that in-situ observations are the only feasible way to search for auroral X-ray emission from Uranus and Neptune.

\footnotetext{
${ }^{9}$ The Chandra observations also revealed X-ray emission coming from Jupiter's moons Io and Europa, and the Io plasma torus (Elsner et al. 2002, 2005b).
} 
Lack of in-situ measurements is not a problem for the last remaining magnetic planet with an atmosphere: the Earth. In contrast to Jupiter's aurora, which is largely powered by energy extracted from planetary rotation, Earth's aurora is generated through the interaction of the solar wind with the magnetosphere (Waite et al. 2001), and its X-ray emission is not caused by charge exchange of energetic heavy ions, but by bremsstrahlung from precipitating electrons (e.g. Bhardwaj 2006). Charge exchange, however, does occur in the Earth exosphere, or geocorona. While this has been known for a long time (Sect. 3.1), its implications for X-ray astronomy were not realized before the discovery of cometary X-rays: Earth is surrounded by an extended X-ray emitting cloud (cf. Fig. 4a), which superimposes a diffuse X-ray glow to observations which are performed from a low Earth orbit. This specific viewing geometry makes it very challenging to disentangle the X-ray emission resulting from charge exchange interactions in the geocorona from other components in the diffuse $\mathrm{X}$-ray sky. For this reason, the implications of charge exchange at Earth are discussed in a separate section.

\subsection{Geocorona and Heliosphere}

As mentioned in Sect. 3.2, CXE produced near the Earth was recorded already in 1990 with Rosat (cf. Fig. 1), but it was either not recognized (comets), or not understood ('long term enhancements') or misinterpreted (Moon) at that time. Soon after the discovery of cometary X-ray emission, however, it became apparent that CXE from the Earth has far-reaching consequences, because the fact that this emission appears to come from larger distances can easily lead to misinterpretations. This problem is not restricted to the geocorona. Also the heliosphere is glowing in X-rays due to CXE. Thus, any X-ray observation made from within the solar system may be affected by charge exchange (e.g. Wargelin et al. 2004).

Soon after the discovery of the importance of charge exchange for the generation of cometary X-rays, it was realized that the same process should also operate at the geocorona (Dennerl et al. 1997; Cox 1998; Freyberg 1998). This was directly demonstrated by Chandra observations of the dark Moon, which revealed the spectral signatures of CXE (Wargelin et al. 2004). Cox (1998) also considered another source of diffuse X-ray emission, due to charge exchange between the solar wind and the stream of interstellar matter flowing through the solar system, causing also the heliosphere to glow in X-rays. There may be yet another source of CXE present which can be attributed to the solar system, caused by instabilities at the heliopause (Borovikov et al. 2008). Compared to the geocoronal component, the heliospheric emission is expected to exhibit slower temporal variations and a different large-scale spatial distribution (Robertson et al. 2001; Robertson and Cravens 2003; Lallement 2004b). A clear distinction between these contributions, however, and especially a determination of their absolute flux, is very difficult, because of the similarity of these components and of the diffuse X-ray emission originating outside the solar system.

The most straightforward method for discriminating the geocoronal and heliospheric CXE from extrasolar X-ray emission is to utilize its temporal variability. In a pioneering study, Snowden et al. (2004) compared four successive XMM-Newton observations of the same part of the sky (the 'Hubble Deep Field North') which does not contain bright X-ray sources, and found that the first three were statistically the same, while the last one exhibited additional flux with a spectral distribution which was consistent with that expected from SWCX. Smith et al. (2005) found evidence for SWCX emission in Chandra observations toward the nearby molecular cloud MBM 12, and Fujimoto et al. (2007) reported the detection of SWCX emission in Suzaku observations of a blank field in the direction of the north ecliptic pole. Carter and Sembay (2008) performed extensive systematic investigations of the XMM-Newton data archive in order to identify observations which were 
affected by SWCX. The most extreme case exhibited a rich emission line spectrum up to $\mathrm{Si} \mathrm{XIV}$ at $2.0 \mathrm{keV}$, indicating that a cloud of plasma associated with a coronal mass ejection had passed the Earth's exosphere (Carter et al. 2010).

While temporal variability studies are capable of identifying periods of enhanced geocoronal and heliospheric X-ray flux, they cannot be used to determine the zero level of the quiescent component. In recent years, the potential implications of solar system CXE for studying extended soft X-ray sources have received increased attention. Charge exchange is now more generally considered as an X-ray 'background' component, no matter whether the data were obtained with XMM-Newton (e.g. Galeazzi et al. 2007; Snowden et al. 2008; Gupta et al. 2009), Chandra (e.g. Smith et al. 2005), or Suzaku (e.g. Smith et al. 2007; Miller et al. 2008; Yoshino et al. 2009). The controversial discussion of a claimed detection of emission from warm-hot gas in the intergalactic medium (Vikhlinin et al. 2001; Arnaud et al. 2001; Kaastra et al. 2003; Finoguenov et al. 2003; Bowyer and Vikhlinin 2004; Bregman and Lloyd-Davies 2006; Nevalainen et al. 2007) illustrates the far-reaching consequences of geocoronal and heliospheric X-rays.

The object which is most severely affected by charge exchange induced X-rays from the solar system is the Local Bubble, an irregularly shaped cavity of $\sim 60$ pc radius around the Sun, supposed to contain a plasma of $\sim 10^{6} \mathrm{~K}$, producing diffuse X-ray emission with spatial and spectral characteristics that are very similar to SWCX. This implies that the quiescent diffuse soft X-ray flux, which was solely attributed to the Local Bubble before the discovery of cometary X-rays, has to be shared with geocoronal and heliospheric CXE (e.g. Cox 1998; Cravens et al. 2001; Lallement 2004b; Henley and Shelton 2008). This new situation has stimulated many efforts to determine how much flux comes from the Local Bubble. In a first attempt, (Cravens 2000) estimated that heliospheric X-ray emission can account for about $25 \%-50 \%$ of the observed soft X-ray background intensities. Koutroumpa et al. found that SWCX can entirely explain the diffuse emission in the $3 / 4 \mathrm{keV}$ band (dominated by O VII and O VIII emission; Koutroumpa et al. 2007), that it contributes significantly in the $1 / 4 \mathrm{keV}$ band (Koutroumpa et al. 2008), and that it can account for most of the observed emission at low galactic latitudes, but not at galactic latitudes above $30^{\circ}$ (Koutroumpa et al. 2009). Robertson et al. (2009) estimated that about half of the soft X-ray background measured during the Rosat all-sky survey is due to emission produced within the heliosphere. Thus, the discovery of SWCX induced X-ray emission requires many previous conclusions and models about the Local Bubble to be revisited (e.g. Shelton 2008, 2009; Lallement 2009).

An interesting possibility to identify the SWCX contribution to the diffuse X-ray flux would be offered by observations with very high spectral resolution, which directly resolve the multiplets of He-like ions, because charge exchange produces characteristic flux ratios there (e.g. Pradhan 1985; Kharchenko et al. 2003; Pepino et al. 2004). ${ }^{10}$ Such observations, with a spectral resolution of $\sim 4 \mathrm{eV}$, have already succeeded with XMM-Newton for Mars (Dennerl et al. 2006). The high spectral resolution here, however, was achieved by slitless X-ray grating spectroscopy (RGS; den Herder et al. 2001), a technique which is not applicable to completely diffuse emission. In this case, the required high spectral resolution could be achieved by a microcalorimeter. McCammon et al. (2002) have already presented a composite spectrum of $\sim 10 \%$ of the sky with a microcalorimeter, where a spectral resolution of $\sim 9 \mathrm{eV}$ was obtained. An upcoming mission with a microcalorimeter will be ASTRO-H, currently scheduled for launch in 2013. Its Soft X-ray Spectrometer (SXS) is expected to

\footnotetext{
${ }^{10}$ However, there might still be a contribution of CXE of extrasolar origin; Sect. 5.1.
} 
provide an energy resolution of 4-7 eV (Mitsuda et al. 2010). An even higher spectral resolution, of $2.5 \mathrm{eV}$, is planned to be obtained with the X-ray Microcalorimeter Spectrometer (XMS) onboard the International X-ray Observatory (IXO; den Herder et al. 2010).

\section{Extrasolar X-rays due to Charge Transfer Reactions}

Although CXE was discovered in the solar system and can be best studied here, observations of this process are not necessarily restricted to the solar system. In recent years, an exciting search has started for evidence of CXE from outside the heliosphere. CXE in the context of extrasolar X-ray studies was already discussed in Sect. 4.4, but there it was considered as a contaminant, while this section will deal with potential sources of extrasolar CXE.

\subsection{Interstellar Medium}

The Interstellar Medium is a very interesting potential location for generating CXE, in particular at interfaces between hot gas and cool clouds. Lallement (2009) noted that, for an $\mathrm{H}$ atom entering a hot plasma, the mean free path against charge transfer is often of the same order as the mean free path against collisional ionization, and that the CXE expected from high velocity clouds in a hot halo is of about the same order as the emission detected with Rosat towards some high velocity clouds (e.g. Kerp et al. 1998).

Lallement (2009) presented evidence for CXE of the North Polar Spur (NPS), an elongated region of extended X-ray emission approximately north of the galactic center, which is already apparent in the uncorrected data from the Rosat all-sky survey (Fig. 1c). The NPS is the brightest filament of the Loop I superbubble, which is known to be the largest coherent structure on the X-ray sky, probably produced by a series of supernovae and stellar winds from the nearby Sco-Cen OB association. Lallement (2009) noticed that the highest quality X-ray spectra of the NPS, obtained with XMM-Newton (Willingale et al. 2003) and Suzaku (Miller et al. 2008), both exhibit a peculiar feature: the emission from the (unresolved) multiplets of the He-like ions O VII and Ne IX is systematically shifted (by $\sim 10-15 \mathrm{eV}$ ) to lower energies relative to the H-like ions O VIII and Ne X. Exactly this behaviour is expected for charge exchange, because this process tends to brighten the flux of the spin forbidden magnetic dipole transition (at $561 \mathrm{eV}$ for O VII) with respect to that of the resonance transition (at $574 \mathrm{eV}$ for O VII), thus causing a systematic shift to lower energies (e.g. Smith et al. 2001; Kharchenko et al. 2003; Pepino et al. 2004). The fact that the energy shift is observed with respect to the emission from the H-like ions O VIII and Ne X (where no energy shift is expected) excludes the possibility that this shift is caused by uncertainties in the absolute energy calibration of the detectors.

Supernova Remnants are another location which contain interfaces between hot gas and cool clouds. As already mentioned in Sect. 3.1, investigations of the role of charge exchange in supernova remnants have a long history (Chamberlain 1956; Serlemitsos et al. 1973). Wise and Sarazin (1989) performed a detailed study of the X-ray emission of supernova remnants which is expected to arise from charge exchange between neutral hydrogen and highly ionized heavy ions in the periphery of a fast shock, and compared this flux with that released by collisional ionization, recombination, and excitation processes between ions and electrons. They found that CXE does not contribute more than $10 \%$ of the emission of the shock heated gas within the remnant, though this emission could be enhanced if unshocked neutral gas would be mixed into the hot shocked gas. They also noted that charge exchange occurs mainly to highly excited states of the ion and can thus produce spectral features 
characteristic of a recombining plasma in what would otherwise be described as an ionizing shock. A more recent investigation by Lallement (2004a) also came to the conclusion that CXE from the interface regions is negligible, although charge exchange could slightly increase the limb brightening if they happen to be oriented along our line of sight.

The role of charge exchange was also investigated in the context of the Galactic ridge $X$-ray emission, originating from a disk-like region around the Galactic center, with a radius of several kpc and a scale height of $\sim 100 \mathrm{pc}$. Tanaka et al. (1999) considered charge exchange interactions between low energy cosmic ray heavy ions with interstellar hydrogen as a possible explanation of the Galactic ridge emission. This process, which had already been investigated earlier for the production of X-ray line emission (Watson 1976; Blint et al. 1976; Christensen et al. 1977, Sect. 3.1), would in general agree with the Suzaku spectra, which show the Galactic ridge emission to be characterized by intense emission lines of Fe and all abundant lower $\mathrm{Z}$ elements (Tanaka 2002). However, this possibility was not supported by more recent observations with Suzaku (Koyama 2007; Ebisawa et al. 2008). As an alternative explanation, (Revnivtsev et al. 2006) suggested that the bulk of the Galactic Ridge emission is composed of weak X-ray sources, mostly cataclysmic variables (White Dwarfs in binary systems, accreting matter from their companions) and coronally active binaries. This was confirmed by an ultradeep $10^{6} \mathrm{~s}$ Chandra observation of a small field near the Galactic Center, where more than $80 \%$ of the Galactic Ridge X-ray emission could be resolved into point sources (Revnivtsev et al. 2009).

\subsection{Stars}

Wargelin and Drake (2001) proposed a novel method of studying stellar winds from late spectral type $(\mathrm{F}-\mathrm{M})$ dwarf stars through searching for CXE signatures. By applying this method to Chandra observations of Proxima Centauri, where no X-ray halo was detected, Wargelin and Drake (2002) could reduce the upper limit on the mass loss rate by orders of magnitude. Based on XMM-Newton observations of the O9.7 Ib supergiant $\zeta$ Orionis, Pollock (2007) suggested that the X-rays do not originate in cooling shocks in the acceleration zone of the stellar wind, but in its terminal velocity regime in collisionless shocks controlled by magnetic fields. He noted that the conditions immediately behind a stellar wind shock are far away from equilibrium, so that charge exchange between ions is likely to be of fundamental importance during the initial phase of the post shock relaxation and may be responsible for the production of many highly charged ions. Pollock (2007) concluded that charge exchange is likely to be as important in stellar winds as it is for the generation of cometary X-rays in the solar wind.

\subsection{Galaxies}

In recent years, indications for CXE have also been detected in the starburst galaxy $M 82$. Tsuru et al. (2007) concluded from a deep observation of M 82 with Suzaku that charge exchange may make a significant contribution to the observed O-K emission in an X-ray bright area of its extended X-ray halo, where the ionized superwind from M 82 can be assumed to collide with cool ambient gas. Evidence for charge exchange processes was also reported by Ranalli et al. (2008) for the central region of M 82, where one of two emission lines seen in XMM-Newton spectra which cannot be accounted for by thermal plasma emission could be explained by CXE from excited O VIII ions. Evidence for CXE has also been found in the bulge of $M 31$ with XMM-Newton, where Liu et al. (2010) have observed an intensity excess from the O VII multiplet. This and the fact that, within the O VII multiplet, the contribution from the spin forbidden transition would be unusually high for pure thermal emission 
(Sect. 5.1), led Liu et al. (2010) to suggest the presence of CXE originating at the interface between the hot gas and a known cool gas spiral.

\subsection{Clusters of Galaxies}

According to Lallement (2004a), the relative importance of CXE compared to thermal emission of a hot plasma, is proportional to the product $v n_{c} n_{e}{ }^{-2}$, where $v$ is the relative velocity between the hot and cold gas, $n_{c}$ the cold gas density, and $n_{e}$ the hot gas density at the interface. Thus, CXE is particularly important when a tenuous hot plasma encounters a dense cold cloud at a high velocity. In this respect, the interaction between cold interstellar clouds and the intracluster gas represents a favorable case for CXE. If there is a significant contribution from charge exchange to the X-ray spectra of very low density gas in galactic halos and intracluster space, then their interpretation in terms of classical thermal plasma emission may be partially misleading, because the observed intensity along the line of sight does not scale with the emission measure, but depends on the characteristics of the interfaces and their number. Thus, at low spectral resolution, CXE can mimic global hot gas cooling. However, this is not global cooling, but instead accelerated cooling restricted to small physical areas, associated with the relative motions of the neutral clouds. Lallement (2004a) concluded that, if CXE is a non negligible contributor to the total X-ray diffuse emission in galaxy clusters, then the cooling strength would be overestimated, and thus the deposited mass. In this way, charge exchange may even be linked to dark matter.

\section{Conclusions and Outlook}

Charge transfer processes are still one of the most important research fields in atomic and molecular physics. This interest stems not only from the fundamental aspects involved, but also from their importance in astrophysical and laboratory plasmas (Chi et al. 2008). The discovery of CXE from comets has had a great impact not only because the intensity of the emission was unexpected but because of the richness of the underlying atomic physics (Otranto et al. 2008). It has opened up an entirely new field of X-ray studies: instead of excitation by hot electrons, these X-rays are produced by ions picking up electrons from neutral gases (Beiersdorfer 2003). After having been overlooked for a long time, the astrophysical importance of CXE is now beginning to receive general attention. Various studies have already spotted a variety of possible astrophysical locations for the generation of CXE, inside (Sect. 4) and outside (Sect. 5) our solar system. While the consequences of considering the presence of CXE are subtle or still speculative in some cases, they are severe in others, including a paradigm change for the Local Bubble.

It is not unlikely that this development, which has just started, will continue, and that evidence for CXE will be found at additional locations. Thus, it may be worthwhile to consider which astrophysical settings are favorable for the generation of CXE. According to Lallement (2004a), these are in particular the interfaces between hot gas and cool clouds, which may be identified by the following criteria:

- tight correlations between $\mathrm{H}_{\alpha}$ and $\mathrm{X}$-ray patterns

- enhanced X-ray limb brightening at interfaces

- spectral and abundance 'anomalies', because CXE spectra differ from thermal spectra and contain only emission lines, resulting in some biases when they are interpreted with classical models. 
A clear discrimination of the characteristic CXE signatures from other sources of X-ray emission, which produce overall similar spectra, requires very high spectral resolution. While such resolution can be achieved already with (slitless) X-ray grating spectroscopy, this technique is only applicable to objects with an extent of less than a few arcminutes. In this context, microcalorimeters will have a high scientific potential, like the Soft X-ray Spectrometer (SXS) onboard ASTRO-H (Mitsuda et al. 2010) or the X-ray Microcalorimeter Spectrometer (XMS) planned for the International X-ray Observatory (IXO). These instruments are expected to provide a non-dispersive spectral resolution of 4-7 eV and $2.5 \mathrm{eV}$.

Considering the potential impact of CXE on various research areas, as indicated by the studies which have already been performed, it will be exciting to follow the future development. This development had started in 1996 with the discovery of cometary X-ray emission. If all the astrophysical implications are considered, one may conclude that this discovery has stimulated a novel look at our universe.

Acknowledgements The author would like to thank the anonymous referee for valuable comments and suggestions.

Open Access This article is distributed under the terms of the Creative Commons Attribution Noncommercial License which permits any noncommercial use, distribution, and reproduction in any medium, provided the original author(s) and source are credited.

\section{References}

R. Ali, C.L. Cocke, M.L.A. Raphaelian, M. Stockli, Multielectron processes in 10-keV/u Ar $q+(5 \leq q \leq 17)$ on Ar collisions. Phys. Rev. A 49, 3586-3596 (1994). doi:10.1103/PhysRevA.49.3586

R.J. Allan, R.E.S. Clegg, A.S. Dickinson, D.R. Flower, Mg-H(+) charge transfer and MG line intensities in gaseous nebulae. Mon. Not. R. Astron. Soc. 235, 1245-1255 (1988)

S.K. Allison, S.D. Warshaw, Passage of heavy particles through matter. Rev. Mod. Phys. 25, $779-817$ (1953). doi:10.1103/RevModPhys.25.779

M. Arnaud, N. Aghanim, R. Gastaud, D.M. Neumann, D. Lumb, U. Briel, B. Altieri, S. Ghizzardi, J. Mittaz, T.P. Sasseen, W.T. Vestrand, XMM-Newton observation of the Coma Galaxy cluster. The temperature structure in the central region. Astron. Astrophys. 365, 67-73 (2001). doi:10.1051/0004-6361:20000195

W. Baade, F. Zwicky, Remarks on super-novae and cosmic rays. Phys. Rev. 46(1), $76-77$ (1934). doi: 10.1103/PhysRev.46.76.2

S.L. Baliunas, S.E. Butler, Silicon lines as spectral diagnostics-The effect of charge transfer. Astrophys. J. 235, 45-48 (1980). doi:10.1086/183154

S.J. Bame, A.J. Hundhausen, J.R. Asbridge, I.B. Strong, Solar wind ion composition. Phys. Rev. Lett. 20, 393-395 (1968). doi:10.1103/PhysRevLett.20.393

S.J. Bame, D.J. McComas, B.L. Barraclough, J.L. Phillips, K.J. Sofaly, J.C. Chavez, B.E. Goldstein, R.K. Sakurai, The ULYSSES solar wind plasma experiment. Astron. Astrophys. Suppl. Ser. 92, 237-265 (1992)

S. Barabash, R. Lundin, T. Zarnowiecki, S. Grzedzielski, Diagnostic of energetic neutral particles at Mars by the ASPERA-C instrument for the Mars 96 mission. Adv. Space Res. 16, 81 (1995). doi:10.1016/0273-1177(95)00212-W

P. Beiersdorfer, Laboratory X-ray astrophysics. Annu. Rev. Astron. Astrophys. 41, 343-390 (2003). doi:10.1146/annurev.astro.41.011802.094825

P. Beiersdorfer, L. Schweikhard, R. Olson, G.V. Brown, S.B. Utter, J.R. Crespo López-Urrutia, K. Widmann, $\mathrm{X}$-ray measurements of charge transfer reactions involving cold, very highly charged ions. Phys. Scr. T 80, 121-123 (1999). doi:10.1238/Physica.Topical.080a00121

P. Beiersdorfer, R.E. Olson, G.V. Brown, H. Chen, C.L. Harris, P.A. Neill, L. Schweikhard, S.B. Utter, K. Widmann, X-ray emission following low-energy charge exchange collisions of highly charged ions. Phys. Rev. Lett. 85(24), 5090-5093 (2000). doi:10.1103/PhysRevLett.85.5090

P. Beiersdorfer, C.M. Lisse, R.E. Olson, G.V. Brown, H. Chen, X-ray velocimetry of solar wind ion impact on comets. Astrophys. J. 549, 47 (2001) 
P. Beiersdorfer, K.R. Boyce, G.V. Brown, H. Chen, S.M. Kahn, R.L. Kelley, M. May, R.E. Olson, F.S. Porter, C.K. Stahle, W.A. Tillotson, Laboratory simulation of charge exchange-produced X-ray emission from comets. Science 300, 1558-1559 (2003)

A. Bhardwaj, X-Ray Emission from Jupiter, Saturn, and Earth: A short review. ArXiv Astrophysics e-prints, 2006

A. Bhardwaj, R.F. Elsner, J.H.J. Waite, G.R. Gladstone, T.E. Cravens, P.G. Ford, Chandra observation of an X-ray flare at Saturn: evidence of direct solar control on Saturn's disk X-ray emissions. Astrophys. J. 624, 121 (2005a)

A. Bhardwaj, G. Branduardi-Raymont, R.F. Elsner, G.R. Gladstone, G. Ramsay, P. Rodriguez, R. Soria, J.H. Waite Jr., T.E. Cravens, Solar control on Jupiter's equatorial X-ray emissions: 26-29 November 2003 XMM-Newton observation. Geophys. Res. Lett. 32, 3 (2005b)

A. Bhardwaj, R.F. Elsner, J.H.J. Waite, G.R. Gladstone, T.E. Cravens, P.G. Ford, The discovery of oxygen $\mathrm{K} \alpha \mathrm{X}$-ray emission from the rings of saturn. Astrophys. J. 627, $73-76$ (2005c). doi:10.1086/431933

A. Bhardwaj, R.F. Elsner, G.R. Gladstone, J.H. Waite, G. Branduardi-Raymont, T.E. Cravens, P.G. Ford, Low- to middle-latitude X-ray emission from Jupiter. J. Geophys. Res. 111(A10), 11225 (2006). doi:10.1029/2006JA011792

A. Bhardwaj, R.F. Elsner, G. Randall Gladstone, T.E. Cravens, C.M. Lisse, K. Dennerl, G. BranduardiRaymont, B.J. Wargelin, J. Hunter Waite, I. Robertson, N. Østgaard, P. Beiersdorfer, S.L. Snowden, V. Kharchenko, X-rays from solar system objects. Planet. Space Sci. 55, 1135-1189 (2007). doi:10.1016/j.pss.2006.11.009

L. Biermann, Kometenschweife und solare Korpuskularstrahlung. Z. Astrophys. 29, 274 (1951)

L. Biermann, Physical processes in comet tails and their relation to solar activity. Phys. Comètes, Extr. Mem. Soc. R. Sci. Liège, 4 Sér. XIII(I-II), 291 (1953)

R. Bingham, J.M. Dawson, V.D. Shapiro, D.A. Mendis, B.J. Kellett, Generation of X-rays from Comet C/Hyakutake 1996 B2. Science 275, 49-51 (1997)

R. Bingham, J.M. Dawson, V.D. Shapiro, D.A. Mendis, B.J. Kellett, Production of X-rays by comet Hyakutake. Adv. Space Res. 22(7), 1043 (1998)

R.J. Blint, W.D. Watson, R.B. Christensen, Calculation of the cross section for C IV-H charge exchange: significance for interstellar X-rays particles. Astrophys. J. 205, 634-637 (1976). doi:10.1086/154320

D. Bodewits, R. Hoekstra, Electron capture in collisions between $\mathrm{O}^{6+}$ ions and $\mathrm{H}_{2} \mathrm{O}$ molecules. Phys. Rev. A 76(3), 032703 (2007). doi:10.1103/PhysRevA.76.032703

D. Bodewits, R. Hoekstra, B. Seredyuk, R.W. McCullough, G.H. Jones, A.G.G.M. Tielens, Charge exchange emission from solar wind helium ions. Astrophys. J. 642, 593-605 (2006). doi:10.1086/500731

D. Bodewits, D.J. Christian, M. Torney, M. Dryer, C.M. Lisse, K. Dennerl, T.H. Zurbuchen, S.J. Wolk, A.G.G.M. Tielens, R. Hoekstra, Spectral analysis of the Chandra comet survey. Astron. Astrophys. 469, 1183-1195 (2007). doi:10.1051/0004-6361:20077410

N. Bohr, K. Lindhard, Electron capture and loss by heavy ions penetrating through matter. K. Dan. Vidensk. Selsk. Mat. Fys. Medd. 28, 1 (1954)

A. Boileau, M. Von Hellermann, L.D. Horton, J. Spence, H.P. Summers, The deduction of low-Z ion temperature and densities in the JET tokamak using charge exchange recombination spectroscopy. Plasma Phys. Control. Fusion 31, 779-804 (1989). doi:10.1088/0741-3335/31/5/006

S.N. Borovikov, N.V. Pogorelov, G.P. Zank, I.A. Kryukov, Consequences of the heliopause instability caused by charge exchange. Astrophys. J. 682, 1404-1415 (2008). doi:10.1086/589634

S. Bowyer, A. Vikhlinin, Detection of emission from warm-hot gas in the Universe with XMM? J. Korean Astron. Soc. 37, 579-581 (2004)

G. Branduardi-Raymont, R.F. Elsner, G.R. Gladstone, G. Ramsay, P. Rodriguez, R. Soria, J.H. Waite, First observation of Jupiter by XMM-Newton. Astron. Astrophys. 424, 331-337 (2004)

G. Branduardi-Raymont, A. Bhardwaj, R.F. Elsner, G.R. Gladstone, G. Ramsay, P. Rodriguez, R. Soria, J.H. Waite Jr., T.E. Cravens, XMM-Newton observations of X-ray emission from Jupiter, in The X-ray Universe 2005, ed. by A. Wilson. ESA Special Publication, vol. 604 (2006), pp. 15-20

G. Branduardi-Raymont, A. Bhardwaj, R.F. Elsner, G.R. Gladstone, G. Ramsay, P. Rodriguez, R. Soria, J.H. Waite Jr., T.E. Cravens, A study of Jupiter's aurorae with XMM-Newton. Astron. Astrophys. 463, 761-774 (2007a). doi:10.1051/0004-6361:20066406

G. Branduardi-Raymont, A. Bhardwaj, R.F. Elsner, G.R. Gladstone, G. Ramsay, P. Rodriguez, R. Soria, J.H. Waite, T.E. Cravens, Latest results on Jovian disk X-rays from XMM-Newton. Planet. Space Sci. 55, 1126-1134 (2007b). doi:10.1016/j.pss.2006.11.017

G. Branduardi-Raymont, R.F. Elsner, M. Galand, D. Grodent, T.E. Cravens, P. Ford, G.R. Gladstone, J.H. Waite, Spectral morphology of the X-ray emission from Jupiter's aurorae. J. Geophys. Res. 113(A12), 2202 (2008). doi:10.1029/2007JA012600

G. Branduardi-Raymont, A. Bhardwaj, R.F. Elsner, P. Rodriguez, X-rays from Saturn: a study with XMMNewton and Chandra over the years 2002-05. Astron. Astrophys. 510(26), 260000 (2010). doi:10.1051/ 0004-6361/200913110 
J.N. Bregman, E.J. Lloyd-Davies, On the lack of a soft X-ray excess from clusters of galaxies. Astrophys. J. 644, 167-173 (2006). doi:10.1086/503551

E.J. Bunce, S.W.H. Cowley, T.K. Yeoman, Jovian cusp processes: implications for the polar aurora. J. Geophys. Res. 109(A18), 9 (2004). doi:10.1029/2003JA010280

R.W. Bussard, R. Ramaty, K. Omidvar, X-ray and gamma-ray line production by nonthermal ions. Astrophys. J. 220, 353-362 (1978). doi:10.1086/155913

C.D. Cantrell, M.O. Scully, K. Boyer, Review of soft X-ray lasers using charge exchange. NASA STI/Recon Technical Report N 75, 27365 (1974)

P.G. Carolan, B.P. Duval, A.R. Field, S.J. Fielding, N.C. Hawkes, N.J. Peacock, G. Fussmann, G. Janeschitz, J. Hofmann, K.H. Behringer, R.C. Isler, Charge-exchange-excited line radiation in a tokamak (ASDEX) with neutral-particle-beam injection. Phys. Rev. A 35, 3454-3471 (1987). doi:10.1103/ PhysRevA.35.3454

J.A. Carter, S. Sembay, Identifying XMM-Newton observations affected by solar wind charge exchange. Part I. Astron. Astrophys. 489, 837-848 (2008). doi:10.1051/0004-6361:200809997

J.A. Carter, S. Sembay, A.M. Read, A high charge state coronal mass ejection seen through solar wind charge exchange emission as detected by XMM-Newton. Mon. Not. R. Astron. Soc. 402, 867-878 (2010). doi:10.1111/j.1365-2966.2009.15985.x

J.W. Chamberlain, Excitation in Nebulae: electron-hydrogen inelastic collisions in a radiative H II region. Astrophys. J. 124, 662 (1956). doi:10.1086/146276

J. Chesnel, B. Sulik, H. Merabet, C. Bedouet, F. Frémont, X. Husson, M. Grether, A. Spieler, N. Stolterfoht, Enhancement of dielectronic processes in $\mathrm{Ne}^{10+}+$ He collisions at low keV energies. Phys. Rev. A 57, 3546-3553 (1998). doi:10.1103/PhysRevA.57.3546

B. Chi, L. Liu, J. Wang, NUCLEAR PHYSICS: Calculations of state-selective differential cross sections for charge transfer in collisions between $\mathrm{O}^{3+}$ and $\mathrm{H}_{2}$. Chin. Phys. B 17, 2890-2896 (2008). doi:10. 1088/1674-1056/17/8/023

R.B. Christensen, W.D. Watson, R.J. Blint, Calculation of the cross section for N IV-H charge-exchange significance for the intercloud gas. Astrophys. J. 213, 712-715 (1977). doi:10.1086/155201

D.J. Christian, D. Bodewits, C.M. Lisse, K. Dennerl, S.J. Wolk, H. Hsieh, T.H. Zurbuchen, L. Zhao, Chandra observations of comets $8 \mathrm{p} /$ Tuttle and $17 \mathrm{p} /$ Holmes during solar minimum. Astrophys. J. Suppl. Ser. 187, 447-459 (2010). doi:10.1088/0067-0049/187/2/447

K.R. Cornelius, K. Wojtkowski, R.E. Olson, State-selective cross section scalings for electron capture collisions. J. Phys. B, At. Mol. Phys. 33, 2017-2035 (2000). doi:10.1088/0953-4075/33/11/304

D.P. Cox, Modeling the local bubble, in Lecture Notes in Physics, vol. 506 (Springer, Berlin, 1998), pp. 121131

T.E. Cravens, Comet Hyakutake X-ray source: Charge transfer of solar wind heavy ions. Geophys. Res. Lett. 24, 105-108 (1997)

T.E. Cravens, Heliospheric X-ray emission associated with charge transfer of the solar wind with interstellar neutrals. Astrophys. J. 532, 153 (2000)

T.E. Cravens, A.N. Maurellis, X-ray emission from scattering and fluorescence of solar X-rays at Venus and Mars. Geophys. Res. Lett. 28(15), 3043-3046 (2001)

T.E. Cravens, E. Howell, J.H. Waite, G.R. Gladstone, Auroral oxygen precipitation at Jupiter. J. Geophys. Res. 100, 17153-17162 (1995). doi:10.1029/95JA00970

T.E. Cravens, I.P. Robertson, S.L. Snowden, Temporal variations of geocoronal and heliospheric X-ray emission associated with the solar wind interaction with neutrals. J. Geophys. Res. 106, 24883-24892 (2001)

T.E. Cravens, J.H. Waite, T.I. Gombosi, N. Lugaz, G.R. Gladstone, B.H. Mauk, R.J. MacDowall, Implications of Jovian X-ray emission for magnetosphere-ionosphere coupling. J. Geophys. Res. 108(A12), 25-1 (2003). doi:10.1029/2003JA010050

T.E. Cravens, J. Clark, A. Bhardwaj, R. Elsner, J.H. Waite, A.N. Maurellis, G.R. Gladstone, G. BranduardiRaymont, X-ray emission from the outer planets: Albedo for scattering and fluorescence of solar X rays. J. Geophys. Res. 111(A10), 7308 (2006). doi:10.1029/2005JA011413

J.A. Crawford, R.P. Kraft, An interpretation of AE Aquarii. Astrophys. J. 123, 44 (1956). doi:10.1086/146128

C.C. Curtis, K.C. Hsieh, Remote sensing of planetary magnetospheres: imaging via energetic neutral atoms, in Outstanding Problems in Solar System Plasma Physics: Theory and Instrumentation (1989), pp. 247251

A. Dalgarno, A. Sternberg, Electron temperatures of astrophysical plasmas. Astrophys. J. 257, 87-90 (1982). doi:10.1086/183814

A. Dalgarno, S.E. Butler, T.G. Heil, Charge transfer of doubly charged oxygen ions in helium. J. Geophys. Res. 85, 6047 (1980). doi:10.1029/JA085iA11p06047

J.W. den Herder, A.C. Brinkman, S.M. Kahn, G. Branduardi-Raymont, K. Thomsen, H. Aarts, M. Audard, J.V. Bixler, A.J. den Boggende, J. Cottam, T. Decker, L. Dubbeldam, C. Erd, H. Goulooze, M. Güdel, P. Guttridge, C.J. Hailey, K.A. Janabi, J.S. Kaastra, P.A.J. de Korte, B.J. van Leeuwen, C. Mauche, 
A.J. McCalden, R. Mewe, A. Naber, F.B. Paerels, J.R. Peterson, A.P. Rasmussen, K. Rees, I. Sakelliou, M. Sako, J. Spodek, M. Stern, T. Tamura, J. Tandy, C.P. de Vries, S. Welch, A. Zehnder, The reflection grating spectrometer on board XMM-Newton. Astron. Astrophys. 365, 7-17 (2001)

J.W. den Herder, R.L. Kelley, K. Mitsuda, L. Piro, S.R. Bandler, P. Bastia, K.R. Boyce, M. Bruin, J.A. Chervenak, L. Colasanti, W.B. Doriese, M. Dipirro, M.E. Eckart, Y. Ezoe, E. Figueroa-Feliciano, L. Ferrari, R. Fujimoto, F. Gatti, K.C. Gendreau, L. Gottardi, R. den Hartog, G.C. Hilton, H. Hoevers, K.D. Irwin, Y. Ishisaki, A. Kashani, C.A. Kilbourne, P. de Korte, J. van der Kuur, C. Macculi, T. Mineo, J.H. Nieland, T. Ohashi, S. Paltani, E. Perinati, F.S. Porter, P.J. Shirron, S.J. Smith, Y. Takei, M. Tashiro, G. Torrioli, M. Tsujimoto, H. van Weers, N.Y. Yamasaki, The X-ray microcalorimeter spectrometer onboard of IXO, in Society of Photo-Optical Instrumentation Engineers (SPIE) Conference Series, vol. 7732 (2010). doi:10.1117/12.856018

K. Dennerl, Discovery of X-rays from Mars with Chandra. Astron. Astrophys. 394, 1119 (2002)

K. Dennerl, X-rays from Mars. Space Sci. Rev. 126, 403-433 (2006). doi:10.1007/s11214-006-9028-7

K. Dennerl, X-rays from Venus observed with Chandra. Planet. Space Sci. 56, 1414-1423 (2008). doi:10.1016/j.pss.2008.03.008

K. Dennerl, X-rays from nonmagnetic planets, in Advances in Geosciences, vol. 15, ed. by A. Bhardwaj et al. (World Scientific, Singapore, 2009), pp. 53-74

K. Dennerl, J. Englhauser, J. Trümper, Comet C/1990 N1 (Tsuchiya-Kiuchi). IAU Circ. 6404 (1996a)

K. Dennerl, J. Englhauser, J. Trümper, X-ray detections of comets. IAU Circ. 6413 (1996b)

K. Dennerl, J. Englhauser, J. Trümper, Comet C/1991 A2 (Arai). IAU Circ. 6472 (1996c)

K. Dennerl, J. Englhauser, J. Truemper, C. Lisse, Comet C/1996 Q1 (Tabur). IAU Circ. 6495 (1996d)

K. Dennerl, J. Englhauser, J. Trümper, X-ray emissions from comets detected in the Röntgen X-ray satellite all-sky survey. Science 277, 1625-1630 (1997)

K. Dennerl, V. Burwitz, J. Englhauser, C. Lisse, S. Wolk, Discovery of X-rays from Venus with Chandra. Astron. Astrophys. 386, 319 (2002)

K. Dennerl, B. Aschenbach, V. Burwitz, J. Englhauser, C.M. Lisse, P.M. Rodriguez-Pascual, A major step in understanding the X-ray generation in comets: recent progress obtained with XMM-Newton, in Society of Photo-Optical Instrumentation Engineers (SPIE) Conference Series, vol. 4851, ed. by J.E. Truemper, H.D. Tananbaum (2003), pp. 277-288. doi:10.1117/12.461137

K. Dennerl, C.M. Lisse, A. Bhardwaj, V. Burwitz, J. Englhauser, H. Gunell, M. Holmström, F. Jansen, V. Kharchenko, P.M. Rodríguez-Pascual, First observation of Mars with XMM-Newton: high resolution X-ray spectroscopy with RGS. Astron. Astrophys. 451, 709 (2006)

K. Dennerl et al. (2011, in preparation)

R. Dörner, V. Mergel, O. Jagutzki, L. Spielberger, J. Ullrich, R. Moshammer, H. Schmidt-Böcking, Cold target recoil ion momentum spectroscopy: a 'momentum microscope' to view atomic collision dynamics. Phys. Rep. 330, 95-192 (2000). doi:10.1016/S0370-1573(99)00109-X

K. Ebisawa, S. Yamauchi, Y. Tanaka, K. Koyama, Y. Ezoe, A. Bamba, M. Kokubun, Y. Hyodo, M. Tsujimoto, H. Takahashi, Spectral study of the galactic ridge X-ray emission with Suzaku. Publ. Astron. Soc. Jpn. 60, 223 (2008)

E. Edgu-Fry, A. Wech, J. Stuhlman, T.G. Lee, C.D. Lin, C.L. Cocke, Cold-target recoil-ion momentum spectroscopy studies of capture from atomic and molecular hydrogen by $\mathrm{O}^{8+}$ and $\mathrm{Ar}^{8+}$. Phys. Rev. A 69(5), 052714 (2004). doi:10.1103/PhysRevA.69.052714

R.F. Elsner, G.R. Gladstone, J.H. Waite, F.J. Crary, R.R. Howell, R.E. Johnson, P.G. Ford, A.E. Metzger, K.C. Hurley, E.D. Feigelson, G.P. Garmire, A. Bhardwaj, D.C. Grodent, T. Majeed, A.F. Tennant, M.C. Weisskopf, Discovery of soft X-ray emission from Io, Europa, and the Io Plasma Torus. Astrophys. J. 572, 1077-1082 (2002)

R.F. Elsner, N. Lugaz, J.H. Waite Jr., T.E. Cravens, G.R. Gladstone, P. Ford, D. Grodent, A. Bhardwaj, R.J. MacDowall, M.D. Desh, T. Majeed, Simultaneous Chandra X ray, Hubble Space Telescope ultraviolet, and Ulysses radio observations of Jupiter's aurora. J. Geophys. Res. 110, 1207 (2005a)

R.F. Elsner, B.D. Ramsey, J.H. Waite, P. Rehak, R.E. Johnson, J.F. Cooper, D.A. Swartz, X-ray probes of magnetospheric interactions with Jupiter's auroral zones, the Galilean satellites, and the Io plasma torus. Icarus 178, 417-428 (2005b). doi:10.1016/j.icarus.2005.06.006

R.C. Elton, R.H. Dixon, J.F. Seely, X-ray laser pumping and charge transfer, in Adaptive Optics and Short Wavelength Sources, ed. by S.F. Jacobs, M. Sargent III, M.O. Scully (1978), pp. 243-265

H.J. Fahr, Charge-transfer interactions between solar wind protons and neutral particles in the vicinity of the Sun. Nature 219, 473-474 (1968). doi:10.1038/219473a0

G.B. Field, G. Steigman, Charge transfer and ionization equilibrium in the interstellar medium. Astrophys. J. 166, 59 (1971). doi:10.1086/150941

A. Finoguenov, U.G. Briel, J.P. Henry, XMM-Newton discovery of an X-ray filament in Coma. Astron. Astrophys. 410, 777-784 (2003). doi:10.1051/0004-6361:20031319 
L.A. Frank, E.L. Ackerson, J.H. Wolfe, J.D. Mihalov, Observations of Plasmas in the Jovian Magnetosphere, 1975

M.J. Freyberg, On the zero-level of the soft X-ray background, in Lecture Notes in Physics, vol. 506 (Springer, Berlin, 1998), pp. 113-116

R. Fujimoto, K. Mitsuda, D. McCammon, Y. Takei, M. Bauer, Y. Ishisaki, S.F. Porter, H. Yamaguchi, K. Hayashida, N.Y. Yamasaki, Evidence for solar-wind charge-exchange X-ray emission from the Earth's magnetosheath. Publ. Astron. Soc. Jpn. 59, 133-140 (2007)

H.O. Funsten, F. Allegrini, G.B. Crew, R. DeMajistre, P.C. Frisch, S.A. Fuselier, M. Gruntman, P. Janzen, D.J. McComas, E. Möbius, B. Randol, D.B. Reisenfeld, E.C. Roelof, N.A. Schwadron, Structures and spectral variations of the outer heliosphere in IBEX energetic neutral atom maps. Science 326, 964 (2009). doi:10.1126/science.1180927

S.A. Fuselier, E.G. Shelley, B.E. Goldstein, R. Goldstein, M. Neugebauer, W.H. Ip, H. Balsiger, H. Rème, Observations of solar wind ion charge exchange in the comet Halley coma. Astrophys. J. 379, 734 (1991)

S.A. Fuselier, F. Allegrini, H.O. Funsten, A.G. Ghielmetti, D. Heirtzler, H. Kucharek, O.W. Lennartsson, D.J. McComas, E. Möbius, T.E. Moore, S.M. Petrinec, L.A. Saul, J.A. Scheer, N. Schwadron, P. Wurz, Width and variation of the ENA flux ribbon observed by the Interstellar Boundary Explorer. Science 326, 962 (2009). doi:10.1126/science. 1180981

M. Galeazzi, A. Gupta, K. Covey, E. Ursino, XMM-Newton observations of the diffuse X-ray background. Astrophys. J. 658, 1081-1087 (2007). doi:10.1086/512032

A.A. Galeev, T.E. Cravens, T.I. Gombosi, Solar wind stagnation near comets. Astrophys. J. 289, 807 (1985)

H. Geiger, E. Marsden, On a diffuse reflection of the $\alpha$-particles. Proc. R. Soc. Lond. Ser. A, Math. Phys. Sci. 82, 495-500 (1909)

R. Giacconi, H. Gursky, F.R. Paolini, Evidence for X rays from sources outside the solar system. Phys. Rev. Lett. 9, 439 (1962)

R. Giacconi, Nobel lecture: The dawn of X-ray astronomy. Rev. Mod. Phys. 75(3), 995 (2003). doi:10.1103/ RevModPhys.75.995

G.R. Gladstone, J.H. Waite, D. Grodent, W.S. Lewis, F.J. Crary, R.F. Elsner, M.C. Weisskopf, T. Majeed, J.M. Jahn, A. Bhardwaj, J.T. Clarke, D.T. Young, M.K. Dougherty, S.A. Espinosa, T.E. Cravens, A pulsating auroral X-ray hot spot on Jupiter. Nature 415, 1000-1003 (2002)

T.I. Gombosi, M. Horanyi, K. Kecskemety, T.E. Cravens, A.F. Nagy, Charge exchange in solar windcometary interactions. Astrophys. J. 268, 889-898 (1983). doi:10.1086/161011

J.B. Greenwood, I.D. Williams, S.J. Smith, A. Chutjian, Measurement of charge exchange and X-ray emission cross sections for solar wind-comet interactions. Astrophys. J. 533, 175 (2000)

J.B. Greenwood, I.D. Williams, S.J. Smith, A. Chutjian, Experimental investigation of the processes determining X-ray emission intensities from charge-exchange collisions. Phys. Rev. A 63(6), 062707 (2001). doi:10.1103/PhysRevA.63.062707

S. Grzedzielski, D. Rucinski, Expected beams of energetic neutral atoms in the outer heliosphere, in Physics of the Outer Heliosphere, ed. by S. Grzedzielski, D.E. Page (1990), pp. 367-370

H. Gunell, E. Kallio, R. Jarvinen, P. Janhunen, M. Holmström, K. Dennerl, Simulations of solar wind charge exchange X-ray emissions at Venus. Geophys. Res. Lett. 34, 3107 (2007). doi:10.1029/2006GL028602

A. Gupta, M. Galeazzi, D. Koutroumpa, R. Smith, R. Lallement, Properties of the diffuse X-ray background toward MBM20 with Suzaku. Astrophys. J. 707, 644-651 (2009). doi:10.1088/0004-637X/707/1/644

J.P. Halpern, J.E. Grindlay, X-ray photoionized nebulae. Astrophys. J. 242, 1041-1055 (1980). doi:10.1086/158535

M. Harwit, F. Hoyle, Plasma dynamics in comets. II. Influence of magnetic fields. Astrophys. J. 135, 875 (1962). doi:10.1086/147331

A.A. Hasan, F. Eissa, R. Ali, D.R. Schultz, P.C. Stancil, State-selective charge transfer studies relevant to solar wind-comet interactions. Astrophys. J. 560, 201 (2001)

L. Haser, Distribution d'intensite dans la tete d'une comete. Bull. Soc. R. Sci. Liège 43, 740-750 (1957)

D.B. Henley, R.L. Shelton, Comparing Suzaku and XMM-Newton observations of the soft X-ray background: evidence for solar wind charge exchange emission. Astrophys. J. 676, 335-350 (2008). doi:10.1086/528924

M. Holmström, S. Barabash, E. Kallio, X-ray imaging of the solar wind-Mars interaction. Geophys. Res. Lett. 28(7), 1287-1290 (2001)

M. Holmström, S. Barabash, E. Kallio, The solar wind interaction with Venus and Mars: Energetic neutral atom and X-ray imaging, in 34th COSPAR Scientific Assembly. COSPAR, Plenary Meeting, vol. 34 (2002)

M. Horanyi, T.E. Cravens, J.H. Waite Jr., The precipitation of energetic heavy ions into the upper atmosphere of Jupiter. J. Geophys. Res. 93, 7251-7271 (1988). doi:10.1029/JA093iA07p07251 
K.C. Hsieh, C.C. Curtis, A model for the spatial and energy distributions of energetic neutral atoms produced within the Saturn/Titan plasma system. Geophys. Res. Lett. 15, $772-775$ (1988). doi:10.1029/GL015i008p00772

K.C. Hsieh, K. Shih, D.J. McComas, S.T. Wu, C.C. Wu, Forecasting the arrival of fast coronal-mass ejecta at Earth by the detection of 2-20 keV neutral atoms, in Society of Photo-Optical Instrumentation Engineers (SPIE) Conference Series, ed. by S. Chakrabarti. Presented at the Society of Photo-Optical Instrumentation Engineers (SPIE) Conference, vol. 1744 (1992a), pp. 72-78

K.C. Hsieh, K.L. Shih, J.R. Jokipii, S. Grzedzielski, Probing the heliosphere with energetic hydrogen atoms. Astrophys. J. 393, 756-763 (1992b). doi:10.1086/171543

H.S. Hudson, W.H. Ip, D.A. Mendis, An Einstein search for X-ray emission from comet Bradfield (19791). Planet. Space Sci. 29, 1373 (1991)

Y. Hui, D.R. Schultz, V.A. Kharchenko, P.C. Stancil, T.E. Cravens, C.M. Lisse, A. Dalgarno, The ion-induced charge-exchange X-ray emission of the Jovian Auroras: magnetospheric or solar wind origin? Astrophys. J. 702, 158-162 (2009). doi:10.1088/0004-637X/702/2/L158

A.J. Hundhausen, H.E. Gilbert, S.J. Bame, The state of ionization of oxygen in the solar wind. Astrophys. J. 152, 3 (1968). doi:10.1086/180165

$\mathrm{S}$. Ibadov, On the efficiency of X-ray generation in impacts of cometary and zodiacal dust particles. Icarus 86, 283-288 (1990)

S. Ibadov, Interplanetary dust interaction with comets: production of X-rays. Adv. Space Res. 17, 1293 (1996)

W. Ip, On the charge exchange loss of energetic charged particles in the Jovian magnetosphere. J. Geophys. Res. 86, 11246-11250 (1981). doi:10.1029/JA086iA13p11246

W.H. Ip, On charge exchange effect in the vicinity of the cometopause of comet Halley. Astrophys. J. 343, 946 (1989)

W.H. Ip, V.W. Chow, NOTE. On Hypervelocity Impact phenomena of microdust and nano X-ray flares in cometary comae. Icarus 130, 217 (1997)

R.C. Isler, An overview of charge-exchange spectroscopy as a plasma diagnostic. Plasma Phys. Control. Fusion 36, 171-208 (1994). doi:10.1088/0741-3335/36/2/001

J.S. Kaastra, R. Lieu, T. Tamura, F.B.S. Paerels, J.W. den Herder, XMM-Newton confirmation of soft Xray excess emission in clusters of galaxies-The discovery of O VII emission from an extended warm baryonic component. Astron. Astrophys. 397, 445-462 (2003). doi:10.1051/0004-6361:20021514

T.R. Kallman, R. McCray, X-ray nebular models. Astrophys. J. Suppl. Ser. 50, 263 (1982)

T.R. Kallman, P. Palmeri, Atomic data for X-ray astrophysics. Rev. Mod. Phys. 79, 79-133 (2007). doi:10.1103/RevModPhys.79.79

E.P. Keath, G.B. Andrews, A.F. Cheng, S.M. Krimigis, B.H. Mauk, D.G. Mitchell, D.J. Williams, Instrumentation for energetic neutral atom imaging of magnetospheres, in Outstanding Problems in Solar System Plasma Physics: Theory and Instrumentation (1989), pp. 165-170

J. Kerp, J. Pietz, P.M.W. Kalberla, W.B. Burton, R. Egger, M.J. Freyberg, D. Hartmann, U. Mebold, Highvelocity clouds and their soft X-ray emission, in IAU Colloq. 166: The Local Bubble and Beyond, ed. by D. Breitschwerdt, M.J. Freyberg, J. Truemper. Lecture Notes in Physics, vol. 506 (Springer, Berlin, 1998), pp. 457-466

V. Kharchenko, Charge-exchange mechanism of X-ray emission, in X-ray Diagnostics of Astrophysical Plasmas: Theory, Experiment, and Observation, vol. 774, ed. by R.K. Smith (2005), p. 271

V. Kharchenko, A. Dalgarno, Spectra of cometary X rays induced by solar wind ions. J. Geophys. Res. 105, 18351-18360 (2000). doi:10.1029/1999JA000203

V. Kharchenko, A. Dalgarno, Variability of cometary X-ray emission induced by solar wind ions. Astrophys. J. 554, 99 (2001)

V. Kharchenko, M. Rigazio, A. Dalgarno, V.A. Krasnopolsky, Charge abundances of the solar wind ions inferred from cometary X-ray spectra. Astrophys. J. 585, 73-75 (2003). doi:10.1086/374209

V. Kharchenko, A. Bhardwaj, A. Dalgarno, D.R. Schultz, P.C. Stancil, Modeling spectra of the north and south Jovian X-ray auroras. J. Geophys. Res. 113(A12), 8229 (2008). doi:10.1029/2008JA013062

E. Kirsch, S.M. Krimigis, W. Ip, G. Gloeckler, X-ray and energetic neutral particle emission from Saturn's magnetosphere. Nature 292, 718-721 (1981). doi:10.1038/292718a0

D. Koutroumpa, F. Acero, R. Lallement, J. Ballet, V. Kharchenko, O VII and O VIII line emission in the diffuse soft X-ray background: heliospheric and galactic contributions. Astron. Astrophys. 475, 901914 (2007). doi:10.1051/0004-6361:20078271

D. Koutroumpa, R. Lallement, V. Kharchenko, A. Dalgarno, The solar wind charge-exchange contribution to the local soft X-ray background. Space Sci. Rev. 87 (2008). doi:10.1007/s11214-008-9381-9

D. Koutroumpa, R. Lallement, J.C. Raymond, V. Kharchenko, The solar wind charge-transfer X-ray emission in the $1 / 4 \mathrm{keV}$ energy range: inferences on local bubble hot gas at low Z. Astrophys. J. 696, 1517-1525 (2009). doi:10.1088/0004-637X/696/2/1517 
K. Koyama, X-ray observations of the Galactic Center with Suzaku. Prog. Theor. Phys. Suppl. 169, 103-108 (2007)

S.S. Krasilnikov, A means of obtaining population inversion at the resonant transition of an ion. Pis ma Z. Tekhn. Fiz. 1, 574-578 (1975)

V. Krasnopolsky, On the nature of soft X-ray radiation in Comets. Icarus 128, 368 (1997)

V.A. Krasnopolsky, Production of cometary X rays by electron impact and Bremsstrahlung processes. J. Geophys. Res. 106, 18741-18750 (2001). doi:10.1029/2001JA000018

V.A. Krasnopolsky, X rays and solar wind composition in four comets observed with Chandra X-Ray Observatory. J. Geophys. Res. 111(A10), 12102 (2006). doi:10.1029/2006JA012003

V.A. Krasnopolsky, M.J. Mumma, Spectroscopy of comet Hyakutake at 80-700 A: first detection of solar wind charge transfer emissions. Astrophys. J. 549, 629 (2001)

V.A. Krasnopolsky, M.J. Mumma, M. Abbott, B.C. Flynn, K.J. Meech, D.K. Yeomans, P.D. Feldman, C.B. Cosmovici, Detection of soft X-rays and a sensitive search for noble gases in comet Hale-Bopp (C/1995 O1). Science 277, 1488-1491 (1997)

V.A. Krasnopolsky, M.J. Mumma, M.J. Abbott, EUVE search for X-rays from comets Encke, Mueller (C/1993 A1), Borrelly, and Postperihelion Hale-Bopp. Icarus 146, 152-160 (2000)

V.A. Krasnopolsky, D.J. Christian, V. Kharchenko, A. Dalgarno, S.J. Wolk, C.M. Lisse, S.A. Stern, X-ray emission from comet McNaught-Hartley (C/1999 T1). Icarus 160, 437-447 (2002)

V.A. Krasnopolsky, J.B. Greenwood, P.C. Stancil, X-ray and extreme ultraviolet emission from comets. Space Sci. Rev. 113, 271 (2004)

S.M. Krimigis, D.G. Mitchell, E.C. Roelof, K.C. Hsieh, D.J. McComas, Imaging the interaction of the heliosphere with the interstellar medium from Saturn with Cassini. Science 326, 971 (2009). doi: $10.1126 /$ science. 1181079

R. Lallement, On the contribution of charge-exchange induced X-ray emission in the ISM and ICM. Astron. Astrophys. 422, 391-400 (2004a). doi:10.1051/0004-6361:20035625

R. Lallement, The heliospheric soft X-ray emission pattern during the ROSAT survey: inferences on local bubble hot gas. Astron. Astrophys. 418, 143-150 (2004b)

R. Lallement, Some observations related to the origin and evolution of the Local Bubble/Local ISM. Space Sci. Rev. 143, 427-436 (2009). doi:10.1007/s11214-008-9428-y

L. Landau, On the theory of stars. Phys. Z. Sowjetunion 1, 285 (1932)

L.J. Lanzerotti, C.G. Maclennan, T.P. Armstrong, S.M. Krimigis, R.P. Lepping, N.F. Ness, Ion and electron angular distributions in the Io torus region of the Jovian magnetosphere. J. Geophys. Res. 86, 8491-8496 (1981). doi:10.1029/JA086iA10p08491

C. Lisse, M. Mumma, R. Petre, K. Dennerl, J. Schmitt, J. Englhauser, J. Trümper, Comet C/1996 B2 (Hyakutake). IAU Circ. 6373 (1996a)

C.M. Lisse, K. Dennerl, J. Englhauser, M. Harden, F.E. Marshall, M.J. Mumma, R. Petre, J.P. Pye, M.J. Ricketts, J. Schmitt, J. Trümper, R.G. West, Discovery of X-ray and extreme ultraviolet emission from comet C/Hyakutake 1996 B2. Science 274, 205-209 (1996b)

C. Lisse, M. Mumma, R. Petre, K. Dennerl, J. Englhauser, J. Schmitt, J. Truemper, Comet C/1996 B2 (Hyakutake). IAU Circ. 6433 (1996c)

C.M. Lisse, D. Christian, K. Dennerl, J. Englhauser, J. Trümper, M. Desch, F.E. Marshall, R. Petre, S. Snowden, X-ray and extreme ultraviolet emission from comet P/Encke 1997. Icarus 141, 316-330 (1999)

C.M. Lisse, D.J. Christian, K. Dennerl, K.J. Meech, R. Petre, H.A. Weaver, S.J. Wolk, Charge exchangeinduced X-ray emission from comet C/1999 S4 (LINEAR). Science 292, 1343-1348 (2001)

C.M. Lisse, T.E. Cravens, K. Dennerl, X-ray and extreme ultraviolet emission from comets. Comets II, 631643 (2004)

J. Liu, Q.D. Wang, Z. Li, J.R. Peterson, X-ray spectroscopy of the hot gas in the M31 bulge. Mon. Not. R. Astron. Soc. 404, 1879-1885 (2010). doi:10.1111/j.1365-2966.2010.16404.x

W.H. Louisell, W.B. McKnight, M.O. Scully, Analysis of a soft-X-ray laser with charge-exchange excitation. Phys. Rev. A 11, 989-1000 (1975). doi:10.1103/PhysRevA.11.989

S. Machida, A. Nishida, Thermal structure of the Jovian plasmasphere. Planet. Space Sci. 26, 745-752 (1978). doi:10.1016/0032-0633(78)90005-3

H.S.W. Massey, E.H.S. Burhop, Electronic and Ionic Impact Phenomena, vol. VIII.6 (Oxford University Press, London, 1952)

H.S.W. Massey, R.A. Smith, The passage of positive ions through gases. Proc. R. Soc. Lond. Ser. A, Math. Phys. Sci. 142, 142-172 (1933)

A.N. Maurellis, T.E. Cravens, G.R. Gladstone, J.H. Waite, L.W. Acton, Jovian X-ray emission from solar X-ray scattering. Geophys. Res. Lett. 27(9), 1339-1342 (2000)

R.J. Mawhorter, A. Chutjian, T.E. Cravens, N. Djurić, S. Hossain, C.M. Lisse, J.A. Macaskill, S.J. Smith, J. Simcic, I.D. Williams, Absolute single and multiple charge exchange cross sections for highly charged C, O, and $\mathrm{Ne}$ ions on $\mathrm{H}_{2} \mathrm{O}, \mathrm{CO}$, and $\mathrm{CO}_{2}$. Phys. Rev. A 75(3), 032704 (2007). doi:10.1103/ PhysRevA.75.032704 
D. McCammon, R. Almy, E. Apodaca, W. Bergmann Tiest, W. Cui, S. Deiker, M. Galeazzi, M. Juda, A. Lesser, T. Mihara, J.P. Morgenthaler, W.T. Sanders, J. Zhang, E. Figueroa-Feliciano, R.L. Kelley, S.H. Moseley, R.F. Mushotzky, F.S. Porter, C.K. Stahle, A.E. Szymkowiak, A high spectral resolution observation of the soft X-ray diffuse background with thermal detectors. Astrophys. J. 576, 188-203 (2002)

D.J. McComas, H.A. Elliott, N.A. Schwadron, J.T. Gosling, R.M. Skoug, B.E. Goldstein, The threedimensional solar wind around solar maximum. Geophys. Res. Lett. 30(10), 100000-1 (2003). doi:10.1029/2003GL017136

D.J. McComas, F. Allegrini, P. Bochsler, M. Bzowski, E.R. Christian, G.B. Crew, R. DeMajistre, H. Fahr, H. Fichtner, P.C. Frisch, H.O. Funsten, S.A. Fuselier, G. Gloeckler, M. Gruntman, J. Heerikhuisen, V. Izmodenov, P. Janzen, P. Knappenberger, S. Krimigis, H. Kucharek, M. Lee, G. Livadiotis, S. Livi, R.J. MacDowall, D. Mitchell, E. Möbius, T. Moore, N.V. Pogorelov, D. Reisenfeld, E. Roelof, L. Saul, N.A. Schwadron, P.W. Valek, R. Vanderspek, P. Wurz, G.P. Zank, Global observations of the interstellar interaction from the Interstellar Boundary Explorer (IBEX). Science 326, 959 (2009). doi:10.1126/science.1180906

A.E. Metzger, D.A. Gilman, J.L. Luthey, K.C. Hurley, H.W. Schnopper, F.D. Seward, J.D. Sullivan, The detection of X-rays from Jupiter. J. Geophys. Res. 88, 7731 (1983)

G. Metzner, Data acquisition and -evaluation electronics for the Rosat PSPCs. Nucl. Instrum. Methods Phys. Res., Sect. A, Accel. Spectrom. Detect. Assoc. Equip. 242, 493-500 (1986). doi:10.1016/ 0168-9002(86)90453-5

E.D. Miller, T. Hiroshi, M.W. Bautz, D. McCammon, R. Fujimoto, J.P. Hughes, S. Katsuda, M. Kokubun, K. Mitsuda, F.S. Porter, Y. Takei, Y. Tsuboi, N.Y. Yamasaki, Suzaku observations of the North Polar Spur: evidence for nitrogen enhancement. Publ. Astron. Soc. Jpn. 60, 95 (2008)

K. Mitsuda, R.L. Kelley, K.R. Boyce, G.V. Brown, E. Costantini, M.J. Dipirro, Y. Ezoe, R. Fujimoto, K.C. Gendreau, J. den Herder, A. Hoshino, Y. Ishisaki, C.A. Kilbourne, S. Kitamoto, D. McCammon, M. Murakami, H. Murakami, M. Ogawa, T. Ohashi, A. Okamoto, S. Paltani, M. Pohl, F.S. Porter, Y. Sato, K. Shinozaki, P.J. Shirron, G.A. Sneiderman, H. Sugita, A. Szymkowiak, Y. Takei, T. Tamagawa, M. Tashiro, Y. Terada, M. Tsujimoto, C. de Vries, H. Yamaguchi, N.Y. Yamasaki, The highresolution X-ray microcalorimeter spectrometer system for the SXS on ASTRO-H, in Society of PhotoOptical Instrumentation Engineers (SPIE) Conference Series, vol. 7732 (2010). doi:10.1117/12.856778

E. Möbius, P. Bochsler, M. Bzowski, G.B. Crew, H.O. Funsten, S.A. Fuselier, A. Ghielmetti, D. Heirtzler, V.V. Izmodenov, M. Kubiak, H. Kucharek, M.A. Lee, T. Leonard, D.J. McComas, L. Petersen, L. Saul, J.A. Scheer, N. Schwadron, M. Witte, P. Wurz, Direct observations of interstellar H, He, and O by the Interstellar Boundary Explorer. Science 326, 969 (2009). doi:10.1126/science.1180971

R.S. Mulliken, Intensities of electronic transitions in molecular spectra II. Charge-transfer spectra. J. Chem. Phys. 7, 20-34 (1939). doi:10.1063/1.1750319

M.J. Mumma, V.A. Krasnopolsky, M.J. Abbott, Soft X-rays from four comets observed with EUVE. Astrophys. J. 491, 125 (1997)

J.U. Ness, J.H.M.M. Schmitt, J. Robrade, Detection of Saturnian X-ray emission with XMM-Newton. Astron. Astrophys. 414, 49-52 (2004a)

J.U. Ness, J.H.M.M. Schmitt, S.J. Wolk, K. Dennerl, V. Burwitz, X-ray emission from Saturn. Astron. Astrophys. 418, 337-345 (2004b)

J. Nevalainen, M. Bonamente, J. Kaastra, Revisiting the soft X-ray excess emission in clusters of galaxies observed with XMM-Newton. Astrophys. J. 656, 733-738 (2007). doi:10.1086/510325

T.G. Northrop, NOTE. The spectrum of X-rays from comet Hyakutake. Icarus 128, 480 (1997)

T.G. Northrop, C.M. Lisse, M.J. Mumma, M.D. Desch, NOTE. A possible source of the X-rays from comet Hyakutake. Icarus 127, 246 (1997)

S. Orsini, I.A. Daglis, M. Candidi, K.C. Hsieh, S. Livi, B. Wilken, Model calculation of energetic neutral atoms precipitation at low altitudes. J. Geophys. Res. 99, 13489 (1994). doi:10.1029/93JA03270

S. Otranto, R.E. Olson, Charge exchange and X-ray emission cross sections for multiply charged ions colliding with $\mathrm{H}_{2}$ O. Phys. Rev. A 77(2), 022709 (2008). doi:10.1103/PhysRevA.77.022709

S. Otranto, R.E. Olson, P. Beiersdorfer, X-ray emission cross sections following charge exchange by multiply charged ions of astrophysical interest. Phys. Rev. A 73(2), 022723 (2006). doi:10.1103/ PhysRevA.73.022723

S. Otranto, R.E. Olson, P. Beiersdorfer, Cometary X-ray emission: theoretical cross sections following charge exchange by multiply charged ions of astrophysical interest. Can. J. Phys. 86, 171-174 (2008). doi:10.1139/P07-127

A. Owens, A.N. Parmar, T. Oosterbroek, A. Orr, L.A. Antonelli, F. Fiore, R. Schulz, G.P. Tozzi, M.C. Maccarone, L. Piro, Evidence for dust-related X-ray emission from comet C/1995 O1 (Hale-Bopp). Astrophys. J. 493, 47 (1998) 
A. Owens, T. Oosterbroek, A. Orr, A.N. Parmar, R. Schulz, L.A. Antonelli, F. Fiore, G.P. Tozzi, M.C. Maccarone, L. Piro, BeppoSAX LECS detection of comet C/1995 O1 (Hale-Bopp). Nucl. Phys. B, Proc. Suppl. 69, 735-738 (1999a). doi:10.1016/S0920-5632(98)00277-1

A. Owens, T. Oosterbroek, A. Orr, A.N. Parmar, R. Schulz, Detection of soft X-ray emission from comet C/1995 O1 (Hale-Bopp). Earth Moon Planets 77, 293-298 (1999b)

R. Pepino, V. Kharchenko, A. Dalgarno, R. Lallement, Spectra of the X-ray emission induced in the interaction between the solar wind and the heliospheric gas. Astrophys. J. 617, 1347 (2004)

D. Pequignot, Charge transfer reactions in some astrophysical situations. Astron. Astrophys. 81, 356-358 (1980a)

D. Pequignot, Charge transfer reactions. II-A photoionization model of the planetary nebula NGC 7662. Astron. Astrophys. 83, 52-57 (1980b)

D. Pequignot, M. Dennefeld, The Crab nebula. Astron. Astrophys. 120, 249-262 (1983)

D. Pequignot, G. Stasińska, S.M.V. Aldrovandi, Charge transfer reactions-A consistent model of the planetary nebula NGC 7027. Astron. Astrophys. 63, 313-324 (1978)

E. Pfeffermann, U. Briel, Development and performance of a position sensitive proportional counter for the ROSAT project. Adv. Space Res. 2(4), 255 (1983)

P.P. Plucinsky, S.L. Snowden, U.G. Briel, G. Hasinger, E. Pfeffermann, An updated calibration of the ROSAT PSPC particle background for the analysis of diffuse and extended sources. Astrophys. J. 418, 519 (1993). doi:10.1086/173414

A.M.T. Pollock, A new paradigm for the X-ray emission of $\mathrm{O}$ stars from XMM-Newton observations of the O9.7 supergiant $\zeta$ Orionis. Astron. Astrophys. 463, 1111-1123 (2007). doi:10.1051/ 0004-6361:20053838

A.K. Pradhan, Recombination-cascade X-ray spectra of highly charged helium-like ions. Astrophys. J. 288, 824 (1985)

L.P. Presniakov, A.P. Shevelko, Intense X-ray emission in the interaction of a laser plasma with a solid surface. ZhETF Pisma Redaktsiiu 36, 38-40 (1982)

P. Ranalli, A. Comastri, L. Origlia, R. Maiolino, A deep X-ray observation of M82 with XMM-Newton. Mon. Not. R. Astron. Soc. 386, 1464-1480 (2008). doi:10.1111/j.1365-2966.2008.13128.x

M. Revnivtsev, S. Sazonov, M. Gilfanov, E. Churazov, R. Sunyaev, Origin of the galactic ridge X-ray emission. Astron. Astrophys. 452, 169-178 (2006). doi:10.1051/0004-6361:20054268

M. Revnivtsev, S. Sazonov, E. Churazov, W. Forman, A. Vikhlinin, R. Sunyaev, Discrete sources as the origin of the galactic X-ray ridge emission. Nature 458, 1142-1144 (2009). doi:10.1038/nature07946

J.E. Rice, E.S. Marmar, J.L. Terry, E. Kaline, J. Kaline, Observation of charge-transfer population of high-n levels in $\mathrm{Ar}^{16+}$ from neutral hydrogen in the ground and excited states in a tokamak plasma. Phys. Rev. Lett. 56, 50-53 (1986). doi:10.1103/PhysRevLett.56.50

M. Rigazio, V. Kharchenko, A. Dalgarno, X-ray emission spectra induced by hydrogenic ions in charge transfer collisions. Phys. Rev. A 66, 064701-1 (2002)

I.P. Robertson, T.E. Cravens, Spatial maps of heliospheric and geocoronal X-ray intensities due to the charge exchange of the solar wind with neutrals. J. Geophys. Res. 108, 6-1 (2003). doi:10.1029/2003JA009873

I.P. Robertson, T.E. Cravens, S. Snowden, T. Linde, Temporal and spatial variations of heliospheric X-ray emissions associated with charge transfer of the solar wind with interstellar neutrals. Space Sci. Rev. 97, 401 (2001)

I.P. Robertson, K.D. Kuntz, M.R. Collier, T.E. Cravens, S.L. Snowden, The heliospheric contribution to the soft X-ray background emission, in American Institute of Physics Conference Series, ed. by R.K. Smith, S.L. Snowden, K.D. Kuntz, vol. 1156 (2009), pp. 52-61. doi:10.1063/1.3211834

E.C. Roelof, Energetic neutral atom image of a storm-time ring current. Geophys. Res. Lett. 14, $652-655$ (1987). doi:10.1029/GL014i006p00652

E.C. Roelof, Imaging heliospheric shocks using energetic neutral atoms, in Solar Wind Seven Colloquium, ed. by E. Marsch, R. Schwenn (1992), pp. 385-390

E.C. Roelof, D.G. Mitchell, D.J. Williams, Energetic neutral atoms $(E \sim 50 \mathrm{keV})$ from the ring current-IMP 7/8 and ISEE 1. J. Geophys. Res. 90, 10991 (1985). doi:10.1029/JA090iA11p10991

E. Rüchardt, Handbuch der Physik, vol. 22.2 (1933)

D.W. Rule, K. Omidvar, Charge equilibrium and radiation of low-energy cosmic rays passing through interstellar medium. Astrophys. J. 229, 1198-1210 (1979). doi:10.1086/157054

E. Rutherford, LXXIX. The scattering of $\alpha$ and $\beta$ particles by matter and the structure of the atom. Philos. Mag., Ser. 6 21.125, 669-688 (1911). doi:10.1080/14786440508637080

H. Ryufuku, K. Sasaki, T. Watanabe, Oscillatory behavior of charge transfer cross sections as a function of the charge of projectiles in low-energy collisions. Phys. Rev. A 21, 745-750 (1980). doi:10.1103/ PhysRevA.21.745

J.H.M.M. Schmitt, S.L. Snowden, B. Aschenbach, G. Hasinger, E. Pfeffermann, P. Predehl, J. Trümper, A soft X-ray image of the Moon. Nature 349, 583 (1991) 
R. Schulz, J.A. Stüwe, G.P. Tozzi, A. Owens, Optical analysis of an activity outburst in comet C/1995 O1 (Hale-Bopp) and its connection to an X-ray outburst. Astron. Astrophys. 361, 359 (2000)

N.A. Schwadron, T.E. Cravens, Implications of solar wind composition for cometary X-rays. Astrophys. J. 544, 558 (2000)

N.A. Schwadron, M. Bzowski, G.B. Crew, M. Gruntman, H. Fahr, H. Fichtner, P.C. Frisch, H.O. Funsten, S. Fuselier, J. Heerikhuisen, V. Izmodenov, H. Kucharek, M. Lee, G. Livadiotis, D.J. McComas, E. Moebius, T. Moore, J. Mukherjee, N.V. Pogorelov, C. Prested, D. Reisenfeld, E. Roelof, G.P. Zank, Comparison of Interstellar Boundary Explorer observations with 3D global heliospheric models. Science 326, 966 (2009). doi:10.1126/science. 1180986

M. Scully, W. Louisell, W. McKnight, A soft X-ray laser utilizing charge exchange. IEEE J. Quantum Electron. 10, 727-728 (1974). doi:10.1109/JQE.1974.1068348

B. Seredyuk, R.W. McCullough, H.B. Gilbody, One-electron capture mechanisms in collisions of Helike $\mathrm{O}$ and $\mathrm{C}$ ions with molecules of astrophysical interest. Phys. Rev. A 72(2), 022710 (2005). doi:10.1103/PhysRevA.72.022710

P.J. Serlemitsos, E.A. Boldt, S.S. Holt, R. Ramaty, A.F. Brisken, X-ray spectrum of Cassiopeia A: evidence for iron line emission. Astrophys. J. 184, 1 (1973). doi:10.1086/181275

V.D. Shapiro, R. Bingham, J.M. Dawson, Z. Dobe, B.J. Kellett, D.A. Mendis, Energetic electrons produced by lower hybrid waves in the cometary environment and soft X-ray emission: Bremsstrahlung and $\mathrm{k}$ shell radiation. J. Geophys. Res. 104, 2537-2554 (1999)

E.G. Shelley, S.A. Fuselier, H. Balsiger, J.F. Drake, J. Geiss, B.E. Goldstein, R. Goldstein, W.H. Ip, A.J. Lazarus, M. Neugebauer, Charge exchange of solar wind ions in the coma of comet P/Halley. Astron. Astrophys. 187, 304 (1987)

R.L. Shelton, The local bubble debate. Space Sci. Rev. 69 (2008). doi:10.1007/s11214-008-9359-7

R.L. Shelton, Revising the local bubble model due to solar wind charge exchange X-ray emission. Space Sci. Rev. 143, 231-239 (2009). doi:10.1007/s11214-008-9358-8

G.A. Shields, A. Dalgarno, A. Sternberg, Line emission from charge transfer with atomic hydrogen at thermal energies. Phys. Rev. A 28, 2137-2140 (1983). doi:10.1103/PhysRevA.28.2137

R.K. Smith, N.S. Brickhouse, D.A. Liedahl, J.C. Raymond, Collisional plasma models with APEC/APED: emission-line diagnostics of hydrogen-like and helium-like ions. Astrophys. J. 556, 91 (2001)

R.K. Smith, R.J. Edgar, P.P. Plucinsky, B.J. Wargelin, P.E. Freeman, B.A. Biller, Chandra observations of MBM 12 and models of the local bubble. Astrophys. J. 623, 225-234 (2005). doi:10.1086/428568

R.K. Smith, M.W. Bautz, R.J. Edgar, R. Fujimoto, K. Hamaguchi, J.P. Hughes, M. Ishida, R. Kelley, C.A. Kilbourne, K.D. Kuntz, D. McCammon, E. Miller, K. Mitsuda, K. Mukai, P.P. Plucinsky, F.S. Porter, S.L. Snowden, Y. Takei, Y. Terada, Y. Tsuboi, N.Y. Yamasaki, Suzaku observations of the local and distant hot ISM. Publ. Astron. Soc. Jpn. 59, 141-150 (2007)

S.L. Snowden, D. McCammon, D.N. Burrows, J.A. Mendenhall, Analysis procedures for ROSAT XRT/PSPC observations of extended objects and the diffuse background. Astrophys. J. 424, 714-728 (1994). doi:10.1086/173925

S.L. Snowden, M.R. Collier, K.D. Kuntz, XMM-Newton observation of solar wind charge exchange emission. Astrophys. J. 610, 1182 (2004)

S.L. Snowden, R.F. Mushotzky, K.D. Kuntz, D.S. Davis, A catalog of galaxy clusters observed by XMMNewton. Astron. Astrophys. 478, 615-658 (2008). doi:10.1051/0004-6361:20077930

A.A. Suchkov, Y.A. Shchekinov, The ion composition of interstellar gas at $10^{4}-10^{5}$ K. Astron. Z. 60, 883 (1983)

Y. Tanaka, ASCA observation of X-ray emission from the Galactic ridge. Astron. Astrophys. 382, 1052-1060 (2002). doi:10.1051/0004-6361:20011678

Y. Tanaka, T. Miyaji, G. Hasinger, Origin of the galactic ridge X-ray emission. Astron. Nachr. 320, 181 (1999)

C.B. Tarter, J.C. Weisheit, A. Dalgarno, Double-charge transfer processes in gaseous nebulae. Astron. Astrophys. 71, 366-368 (1979)

M. Torney, R. Bingham, J.M. Dawson, B.J. Kellett, V.D. Shapiro, H. Summers, Modelling Xray line and continuum emission from comets. Phys. Scr. T 98, 168-171 (2001). doi:10.1238/ Physica.Topical.098a00168

J. Trümper, The ROSAT mission. Adv. Space Res. 2, 241-249 (1982). doi:10.1016/02731177(82)90070-9

T.G. Tsuru, M. Ozawa, Y. Hyodo, H. Matsumoto, K. Koyama, H. Awaki, R. Fujimoto, R. Griffiths, C. Kilbourne, K. Matsushita, K. Mitsuda, A. Ptak, P. Ranalli, N.Y. Yamasaki, X-ray spectral study of the extended emission, 'the cap', located $11.6 \mathrm{kpc}$ above the disk of M82. Publ. Astron. Soc. Jpn. 59, 269282 (2007)

W.H. Tucker, R.J. Gould, Radiation from a low-density plasma at $10^{6} \mathrm{deg}-10^{8} \mathrm{deg}$ K. Astrophys. J. 144, 244 (1966). doi:10.1086/148601 
M. Uchida, M. Morikawa, H. Kubotani, H. Mouri, X-ray spectra of comets. Astrophys. J. 498, 863 (1998)

A. Vikhlinin, M. Markevitch, W. Forman, C. Jones, Zooming in on the coma cluster with Chandra: compressed warm gas in the brightest cluster galaxies. Astrophys. J. 555, 87-90 (2001). doi:10.1086/323181

W. Voges, B. Aschenbach, T. Boller, H. Brauninger, U. Briel, W. Burkert, K. Dennerl, J. Englhauser, R. Gruber, F. Haberl, G. Hartner, G. Hasinger, E. Pfeffermann, W. Pietsch, P. Predehl, J. Schmitt, J. Trumper, U. Zimmermann, Rosat all-sky survey faint source catalogue. IAU Circ. 7432 (2000)

J.H. Waite Jr., F. Bagenal, F. Seward, C. Na, G.R. Gladstone, T.E. Cravens, K.C. Hurley, J.T. Clarke, R. Elsner, S.A. Stern, ROSAT observations of the Jupiter aurora. J. Geophys. Res. 99, 14799 (1994). doi:10.1029/94JA01005

J.H. Waite, G.R. Gladstone, W.S. Lewis, P. Drossart, T.E. Cravens, A.N. Maurellis, B.H. Mauk, S. Miller, Equatorial X-ray emissions: implications for Jupiter's high exospheric temperatures. Science 276, 104108 (1997). doi:10.1126/science.276.5309.104

J.H. Waite, G.R. Gladstone, W.S. Lewis, R. Goldstein, D.J. McComas, P. Riley, R.J. Walker, P. Robertson, S. Desai, J.T. Clarke, D.T. Young, An auroral flare at Jupiter. Nature 410, 787-789 (2001)

M.K. Wallis, Weakly-shocked flows of the solar wind plasma through atmospheres of comets and planets. Planet. Space Sci. 21, 1647-1660 (1973). doi:10.1016/0032-0633(73)90156-6

B.J. Wargelin, J.J. Drake, Observability of stellar winds from late-type dwarfs via charge exchange X-ray emission. Astrophys. J. 546, 57 (2001)

B.J. Wargelin, J.J. Drake, Stringent X-ray constraints on mass loss from Proxima Centauri. Astrophys. J. 578, 503-514 (2002). doi:10.1086/342270

B.J. Wargelin, M. Markevitch, M. Juda, V. Kharchenko, R. Edgar, A. Dalgarno, Chandra observations of the "dark" Moon and geocoronal solar wind charge transfer. Astrophys. J. 607, 596 (2004)

B.J. Wargelin, P. Beiersdorfer, P.A. Neill, R.E. Olson, J.H. Scofield, Charge-exchange spectra of hydrogenic and He-like iron. Astrophys. J. 634, 687-697 (2005). doi:10.1086/496874

B.J. Wargelin, P. Beiersdorfer, G.V. Brown, EBIT charge-exchange measurements and astrophysical applications. Can. J. Phys. 86, 151-169 (2008). doi:10.1139/P07-125

W.D. Watson, Production of galactic X-rays following charge exchange by cosmic-ray nuclei. Astrophys. J. 206, 842-846 (1976). doi:10.1086/154447

R. Wegmann, K. Dennerl, X-ray tomography of a cometary bow shock. Astron. Astrophys. 430, 33-36 (2005)

R. Wegmann, H.U. Schmidt, C.M. Lisse, K. Dennerl, J. Englhauser, X-rays from comets generated by energetic solar wind particles. Planet. Space Sci. 46.5, 603 (1998)

R. Wegmann, K. Dennerl, C.M. Lisse, The morphology of cometary X-ray emission. Astron. Astrophys. 428, 647-661 (2004)

N.C. Wickramasinghe, F. Hoyle, Very small dust particles (VDSP's) in comet C/1996 B2 (Hyakutake). Astrophys. J. Suppl. Ser. 239, 121 (1996)

R.E. Williams, The ionization structure of planetary nebulae-X. The contribution of charge exchange and optically thick condensations to [O I] radiation. Mon. Not. R. Astron. Soc. 164, 111-119 (1973)

R. Willingale, A.D.P. Hands, R.S. Warwick, S.L. Snowden, D.N. Burrows, The X-ray spectrum of the North Polar Spur. Mon. Not. R. Astron. Soc. 343, 995-1001 (2003). doi:10.1046/j.1365-8711.2003.06741.x

M.W. Wise, C.L. Sarazin, Charge transfer and X-ray emission from supernova remnants. Astrophys. J. 345, 384-392 (1989). doi:10.1086/167913

T. Yoshino, K. Mitsuda, N.Y. Yamasaki, Y. Takei, T. Hagihara, K. Masui, M. Bauer, D. McCammon, R. Fujimoto, Q.D. Wang, Y. Yao, Energy spectra of the soft X-ray diffuse emission in fourteen fields observed with Suzaku. Publ. Astron. Soc. Jpn. 61, 805 (2009) 